University of Nebraska - Lincoln

DigitalCommons@University of Nebraska - Lincoln

$11-1-2003$

\title{
Effect of external electric field on the bulk and interfacial properties of weakly dipolar fluid in slab-shaped and sphere- shaped systems
}

\author{
V.B. Warshavsky \\ University of Nebraska-Lincoln \\ Xiao Cheng Zeng \\ University of Nebraska-Lincoln, xzeng1@unl.edu
}

Follow this and additional works at: https://digitalcommons.unl.edu/chemzeng

Part of the Chemistry Commons

Warshavsky, V.B. and Zeng, Xiao Cheng, "Effect of external electric field on the bulk and interfacial properties of weakly dipolar fluid in slab-shaped and sphere-shaped systems" (2003). Xiao Cheng Zeng Publications. 30.

https://digitalcommons.unl.edu/chemzeng/30

This Article is brought to you for free and open access by the Published Research - Department of Chemistry at DigitalCommons@University of Nebraska - Lincoln. It has been accepted for inclusion in Xiao Cheng Zeng Publications by an authorized administrator of DigitalCommons@University of Nebraska - Lincoln. 


\title{
Effect of external electric field on the bulk and interfacial properties of weakly dipolar fluid in slab-shaped and sphere-shaped systems
}

\author{
V. B. Warshavsky and X. C. Zeng \\ Department of Chemistry, University of Nebraska-Lincoln, Lincoln, Nebraska 68588, USA
}

(Received 25 June 2003; published 24 November 2003)

\begin{abstract}
The effect of a uniform electric field on the bulk and interfacial properties of a model dipolar fluid is investigated by using a modified mean-field density functional theory. Particular attention is given to the dependence of the vapor-liquid phase coexistence in a slab-shaped system on the direction of the electric field with respect to the slab surfaces, as well as in the sphere-shaped system on the surrounding dielectric permittivity. For planar vapor-liquid interfaces, the interfacial profiles of the orientation order parameters and components of the dielectric-permittivity tensor are calculated. Analytical expressions for these interfacial profiles and their dependence on the electric field are obtained. When the electric field is normal to the interface we find that the thermodynamic surface tension is lowered compared to that in zero field, and that when the electric field is parallel to the interface the surface tension is enhanced. In contrast, the mechanical surface tension at the equimolar dividing surface is always enhanced by the field regardless of the field direction, and it assumes its highest value when the field is parallel to the interface.
\end{abstract}

DOI: 10.1103/PhysRevE.68.051203

PACS number(s): 68.03. $-\mathrm{g}$, 82.60.Nh, 92.60.Jq, 92.60.Ls

\section{INTRODUCTION}

In two previous papers [1,2], we studied the effect of a uniform electric field on the interfacial properties of a model dipolar and dipolar-quadrupolar fluid on the basis of a modified mean-field density functional theory $[3,4]$. In both papers, we considered only a special case for the system, that is, the direction of the field is perpendicular to the planar vapor-liquid interface. For the dipolar system, we found that the electric field lowers the thermodynamic surface tension but enhances the mechanical surface tension at the equimolar dividing surface. We gave an estimation that a field as high as $10^{8} \mathrm{~V} / \mathrm{m}$ may be required in order to detect the field effect on the thermodynamic and mechanical surface tensions. In this work, we consider a more general case, that is, the electric field can have an arbitrary direction with respect to the vapor-liquid interface. We pay particular attention to the vapor-liquid phase coexistence in both slab-shaped and sphere-shaped systems and their dependence on the direction of the field as well as on the surrounding dielectric permittivity.

Knowledge of the field effect on the surface tension is of importance to the study of liquid droplet nucleation in the electric field [2]. This is because the rate of nucleation depends exponentially on the surface tension, according to the classical theory of nucleation, and thus a small change in the surface tension can result in huge changes in the rate of nucleation. A better understanding of the field effect on the vapor-liquid interfacial properties is also useful for the ellipsometry measurement of the width of the interface, as the measurement requires knowledge of the dielectricpermittivity profile across the interface. The dielectricpermittivity profile essentially describes the response of an inhomogeneous dielectric to external electric fields $[5,6]$.

A number of theoretical methods have been developed toward understanding the interfacial properties of dipolar fluids in the electric field. These properties include the orienta- tion of molecular ordering $[7,8]$, the dielectric-permittivity profiles $[9,10]$, and the surface tensions [11-13]. Among many theoretical methods developed, density functional theory (DFT) is one of the most successful for studying vapor-liquid interfaces [3,14-16]. The DFT allows both the structural (the density and orientation profiles) and thermodynamic (surface tension) properties to be determined in a self-consistent fashion. For weakly dipolar fluids, the modified mean-field (MMF) DFT developed by Teixeira and Telo da Gama $[3,4]$ has been employed by many researchers including us [1,17-21], although more sophisticated DFT has also been developed for strongly dipolar fluids [16,22,23].

The paper is organized as follow. In Sec. II the MMF DFT is extended to include the contribution due to the electric field with an arbitrary direction with respect to the interface. In Sec. III we study the field dependence of the bulk properties and vapor-liquid phase coexistence. In Sec. IV, we present results for the density and dielectric-permittivity profiles, the surface tension as well as their field dependence. Conclusions are given in Sec. V.

\section{MODIFIED MEAN-FIELD DENSITY FUNCTIONAL THEORY FOR WEAKLY DIPOLAR FLUIDS}

We consider a single-component dipolar fluid whose density is given by $\rho(\vec{r}, \omega)$ where $\vec{r}$ denotes the location and $\omega$ the orientation of the molecular dipole. The number density is written as

$$
\rho(\vec{r}, \omega)=\rho(\vec{r}) \hat{f}(\vec{r}, \omega),
$$

where $\rho(\vec{r})$ is the number density of molecules without specifying orientation and $\hat{f}(\vec{r}, \omega)$ is the orientation distribution function that satisfies the normalization condition $\int d \omega \hat{f}(\vec{r}, \omega)=1$. The pairwise potential between the molecules $u\left(\vec{r}_{1}, \vec{r}_{2}, \omega_{1}, \omega_{2}\right)$ is divided into a short-ranged repulsive part $u_{r e f}\left(\vec{r}_{1}, \vec{r}_{2}, \omega_{1}, \omega_{2}\right)$ and a long-ranged attractive 
part $u_{p e r}\left(\vec{r}_{1}, \vec{r}_{2}, \omega_{1}, \omega_{2}\right)$. The former is treated as the reference and the latter is treated as a perturbation. Here, the reference and perturbation potentials [3] are given by

$$
u_{r e f}\left(r_{12}\right)= \begin{cases}+\infty, & r_{12} \leqslant d \\ 0, & r_{12}>d\end{cases}
$$

where $r_{12}=\left|\vec{r}_{2}-\vec{r}_{1}\right|$ is the intermolecular distance, $d$ the hard-sphere diameter, and

$$
\begin{aligned}
u_{\text {per }}\left(\vec{r}_{12}, \omega_{1}, \omega_{2}\right)= & {\left[-4 \epsilon\left(d / r_{12}\right)^{6}+u_{d d}\left(\vec{r}_{12}, \omega_{1}, \omega_{2}\right)\right] } \\
& \times H\left(r_{12}-d\right) .
\end{aligned}
$$

In Eq. (3) $\epsilon$ is the energy parameter of the isotropic Sutherland potential [24,25], $H(r)$ the Heaviside step function, and $u_{d d}$ the interaction between dipoles with an identical moment $\mu_{0}$, i.e.,

$$
\begin{aligned}
u_{d d}\left(\vec{r}_{12}, \omega_{1}, \omega_{2}\right)= & -\left\{3\left[\vec{\mu}_{0}\left(\omega_{1}\right) \cdot \vec{e}_{r}\right]\left[\vec{\mu}_{0}\left(\omega_{2}\right) \cdot \vec{e}_{r}\right]\right. \\
& \left.-\left[\vec{\mu}_{0}\left(\omega_{1}\right) \cdot \vec{\mu}_{0}\left(\omega_{2}\right)\right]\right\} / r_{12}^{3},
\end{aligned}
$$

where $\vec{\mu}_{0}(\omega)$ denotes the dipole vector and $\vec{e}_{r}=\vec{r}_{12} / r_{12}$ is the unit vector connecting molecules 1 and 2 .

In the framework of DFT $[3,16]$, the grand potential of the dipolar fluid can be expressed as

$$
\begin{aligned}
\Omega[\rho(\vec{r}, \omega)]= & \int_{V} d \vec{r} f_{h s}(\rho(\vec{r}))+(1 / \beta) S+\Omega_{i n t} \\
& -\mu \int_{V} d \omega d \vec{r} \rho(\vec{r}, \omega)+\Omega_{E}
\end{aligned}
$$

where $V$ is the volume of the system, $\mu$ is the chemical potential of the fluid, $\beta=1 /\left(k_{B} T\right)$ ( $k_{B}$ the Boltzmann constant, $T$ the temperature), and $f_{h s}(\rho(\vec{r}))$ is the free energy density of the hard-sphere reference system, which can be calculated via the Carnahan-Starling formula [26]. The term

$$
S=\int_{V} d \vec{r} \rho(\vec{r})\langle\ln [4 \pi \hat{f}(\vec{r}, \omega)]\rangle
$$

accounts for the loss of entropy in the reference system due to the orientational degrees of freedom, and $\langle\cdots\rangle$ $\equiv \int \cdots \hat{f}(\vec{r}, \omega) d \omega$ denotes the orientational average weighted by the orientation distribution function. The term $\Omega_{i n t}$ describes the contribution due to the perturbation potential $u_{p e r}$. A commonly used approximation to the pair correlation function that appears in the expression for $\Omega_{\text {int }}$ is the modified mean-field approximation $[3,16,17]$, which describes the correlation function by a Boltzmann term. Following previous work $[3,17]$, we expand the exponential term (in $\left.\Omega_{\text {int }}\right)$ in powers of $\beta u_{\text {per }}\left(\vec{r}_{12}, \omega_{1}, \omega_{2}\right)$ and keep terms up to the second order:

$$
\begin{aligned}
\Omega_{i n t}= & \frac{1}{2 \beta} \int_{V} d \vec{r}_{1} \int_{V} d \vec{r}_{2} \iint d \omega_{1} d \omega_{2} \rho\left(\vec{r}_{1}, \omega_{1}\right) \rho\left(\vec{r}_{2}, \omega_{2}\right) \\
& \times e^{-\beta u_{r e f}\left(r_{12}\right)}\left(u_{p e r}-\frac{1}{2 \beta} u_{p e r}^{2}\right)
\end{aligned}
$$

Such a perturbation approach is applicable only to weakly dipolar systems. For example, when compared with the nonperturbation approach [16,22], Frodl and Dietrich have shown that the discrepancy in the predicted critical temperature is less than $10 \%$ if the reduced dipole moment (defined later) is less than 1. Note that $\Omega_{\text {int }}$ is the only term in Eq. (5) that depends on the geometry of the dielectric system, for it is related to $u_{p e r}$. Lastly, the term $\Omega_{E}$ in Eq. (5) stems from the energy contribution due to the presence of the external field $\vec{E}$, i.e.,

$$
\Omega_{E}=-\int_{V} d \vec{r} \int d \omega \rho(\vec{r}, \omega)\left[\vec{\mu}_{0}(\omega) \cdot \vec{E}\right]
$$

Consider that the planar interfacial system is enclosed in an infinite slab. The planar interface with an area $A$ is in parallel with the surfaces of the slab and in the $x-y$ plane. A uniform electric field $\vec{E}$ parallel to the $x-z$ plane is applied onto the slab system and thus it has two components, $\vec{E}=\left(E_{\|}, E_{\perp}\right)$, where $E_{\|}$and $E_{\perp}$ are the components in the $x$ and $z$ directions, respectively. As such, the system is inhomogeneous only in the $z$ direction. Consequently, the number density $\rho(z)$ as well as the orientation distribution $\hat{f}(z, \omega)$ exhibit only $z$ dependence for the spatial variables. The solid angle $\omega=(\theta, \varphi)$ describes the orientation of the dipole vector $\vec{\mu}_{0}(\omega)$ in the space-fixed coordinates. Under these conditions, we substitute Eqs. (1)-(4) into Eq. (7) and integrate out the $x$ and $y$ spatial variables. $\Omega_{\text {int }}$ can then be written as an integral $[2,3]$

$$
\frac{\Omega_{\text {int }}}{A}=\frac{1}{2} \int_{-\infty}^{\infty} \int_{-\infty}^{\infty} d z_{1} d z_{2} \rho\left(z_{1}\right) \rho\left(z_{2}\right) k\left(z_{1}, z_{2}\right)
$$

with the kernel

$$
k\left(z_{1}, z_{2}\right)=\int d \omega_{1} d \omega_{2} \hat{f}\left(z_{1}, \omega_{1}\right) \hat{f}\left(z_{2}, \omega_{2}\right) \phi_{e f f}\left(z_{12}, \omega_{1}, \omega_{2}\right),
$$

where $z_{12}=z_{2}-z_{1}$. The explicit form of $\phi_{\text {eff }}\left(z_{1}, z_{2}\right)$ is given in the Appendix.

The orientation distribution $\hat{f}(z, \omega)$ can be written as a sum of the isotropic part $1 / 4 \pi$ and a small anisotropic correction $\Delta \hat{f}(z, \omega)$ due to dipole-dipole and dipole-field interactions, i.e.,

$$
\hat{f}(z, \omega)=\frac{1}{4 \pi}+\Delta \hat{f}(z, \omega), \quad(\Delta \hat{f} / \hat{f} \ll 1) .
$$

Note that for isotropic molecular fluids $\Delta \hat{f}=0$. With Eq. (11) $S$ in Eq. (6) can be reduced to [17] 


$$
\frac{S}{A}=2 \pi \int_{-\infty}^{+\infty} d z \rho(z) \int d \omega|\Delta \hat{f}(z, \omega)|^{2} .
$$

The anisotropic part of $\Delta \hat{f}(z, \omega)$ can be expressed as an expansion in terms of spherical harmonics $Y_{l m}(\omega)$ with the expansion coefficients $\left\{\eta_{l m}(z)\right\}$ as the orientation order parameters,

$$
\begin{aligned}
\Delta \hat{f}(z, \omega)= & \sum_{l=1}^{2} \sum_{m=-l}^{l}\left[\frac{(2 l+1)}{4 \pi}\right]^{1 / 2}\left[\frac{(l-|m|) !}{(l+|m|) !}\right]^{1 / 2} \\
& \times \eta_{l, m}(z) Y_{l m}(\omega), \\
\eta_{l, m}(z)= & {\left[\frac{4 \pi}{2 l+1}\right]^{1 / 2}\left[\frac{(l+|m|) !}{(l-|m|) !}\right]^{1 / 2}\left\langle Y_{l m}^{*}(\omega)\right\rangle } \\
& (l=1,2 ;|m| \leqslant l) .
\end{aligned}
$$

Because $\hat{f}(z, \omega)$ is real, we have $\eta_{l, m}(z)=(-1)^{m} \eta_{l,-m}(z)$. Following previous work $[3,14,16]$, we truncate the expansion in Eq. (13) at $l=2$ because a major contribution to the anisotropic orientation of the dipolar molecules at the vaporliquid interface arises from the second-order term. Hereafter, the three order parameters for $l=1, \eta_{1,0}(z), \eta_{1,-1}(z)$, and $\eta_{1,1}(z), \quad$ are denoted as $\eta_{\perp}(z)=\eta_{1,0}(z)$ and $\eta_{\|}(z)$ $=\eta_{1,-1}(z)=-\eta_{1,1}(z)$.

With the above equations and using the orthogonality condition for the spherical harmonics, Eq. (5) can be rewritten as a functional of $\rho(z)$ and $\left\{\eta_{l m}(z)\right\}$, i.e.,

$$
\begin{aligned}
& \Omega\left[\rho\left(z_{1}\right),\left\{\eta_{l, m}\left(z_{1}\right)\right\}\right] \\
& =\int_{-\infty}^{\infty} d z_{1} f_{h s}\left(\rho\left(z_{1}\right)\right)+\frac{1}{\beta} \int_{-\infty}^{\infty} d z_{1} \rho\left(z_{1}\right)\left\{\frac{3}{2} \eta_{\perp}^{2}\left(z_{1}\right)\right. \\
& \left.\quad+\frac{3}{2} \eta_{\|}^{2}\left(z_{1}\right)+\frac{5}{2} \eta_{2,0}^{2}\left(z_{1}\right)+\frac{5}{6} \eta_{2,1}^{2}\left(z_{1}\right)+\frac{5}{24} \eta_{2,2}^{2}\left(z_{1}\right)\right\} \\
& \quad+\int_{-\infty}^{\infty} \int_{-\infty}^{\infty} d z_{1} d z_{2} \rho\left(z_{1}\right) \rho\left(z_{2}\right) k\left(z_{1}, z_{2}\right) \\
& \quad-\mu \int_{-\infty}^{\infty} d z_{1} \rho\left(z_{1}\right)-\mu_{0} \int_{-\infty}^{\infty} d z_{1} \rho\left(z_{1}\right) \\
& \quad \times\left[E_{\perp} \eta_{\perp}\left(z_{1}\right)+E_{\|} \eta_{\|}\left(z_{1}\right)\right],
\end{aligned}
$$

where

$$
\begin{aligned}
k\left(z_{1}, z_{2}\right)= & \phi_{0}\left(z_{12}\right)-\eta_{2,0}\left(z_{1}\right) \eta_{2,0}\left(z_{2}\right) V\left(z_{12}\right)-\left[\eta_{2,0}\left(z_{1}\right)\right. \\
& \left.+\eta_{2,0}\left(z_{2}\right)\right] \phi_{2}\left(z_{12}\right)-\left[\eta_{\perp}\left(z_{1}\right) \eta_{\perp}\left(z_{2}\right)\right. \\
& \left.-\eta_{\|}\left(z_{1}\right) \eta_{\|}\left(z_{2}\right) / 2\right] \phi_{1}\left(z_{12}\right),
\end{aligned}
$$

and where the functions $\phi_{0}(z), \phi_{1}(z), \phi_{2}(z)$, and $V(z)$ are given in Eq. (A15) of Ref. [3].

At a given temperature and chemical potential, the equilibrium density and orientation order-parameter profiles are determined by functional minimization of the grand canonical potential with respect to $\rho(z)$ and $\left\{\eta_{l m}(z)\right\}$, i.e.,

$$
\begin{aligned}
\mu= & \mu_{h s}\left(\rho\left(z_{1}\right)\right)+\frac{3}{2 \beta} \eta_{\perp}^{2}\left(z_{1}\right)+\frac{3}{2 \beta} \eta_{\|}^{2}\left(z_{1}\right)+\frac{5}{2 \beta} \eta_{2,0}^{2}\left(z_{1}\right) \\
& +\int_{-\infty}^{\infty} d z_{2} k\left(z_{1}, z_{2}\right) \rho\left(z_{2}\right)-\mu_{0}\left[E_{\perp} \eta_{\perp}\left(z_{1}\right)+E_{\|} \eta_{\|}\left(z_{1}\right)\right],
\end{aligned}
$$

where $\mu_{h s}\left(\rho\left(z_{1}\right)\right)=\partial f_{h s}\left(\rho\left(z_{1}\right)\right) / \partial \rho\left(z_{1}\right)$ is the local chemical potential of the hard-sphere fluid, and

$$
\begin{gathered}
\eta_{\perp}\left(z_{1}\right)=\frac{\beta \mu_{0} E_{\perp}}{3}+\frac{\beta}{3} \int_{-\infty}^{\infty} d z_{2} \rho\left(z_{2}\right) \eta_{\perp}\left(z_{2}\right) \phi_{1}\left(z_{12}\right), \\
\eta_{\|}\left(z_{1}\right)=\frac{\beta \mu_{0} E_{\|}}{3}-\frac{\beta}{6} \int_{-\infty}^{\infty} d z_{2} \rho\left(z_{2}\right) \eta_{\|}\left(z_{2}\right) \phi_{1}\left(z_{12}\right), \\
\eta_{2,0}\left(z_{1}\right)=\frac{\beta}{5} \int_{-\infty}^{\infty} d z_{2} \rho\left(z_{2}\right)\left[\eta_{2,0}\left(z_{2}\right) V\left(z_{12}\right)+\phi_{2}\left(z_{12}\right)\right] .
\end{gathered}
$$

Because the $l=2$ order parameters $\eta_{2,1}\left(z_{1}\right)$ and $\eta_{2,2}\left(z_{1}\right)$ are incorporated only in the orientation entropy term in the expression for $\Omega$, the variation of $\Omega$ with respect to $\eta_{2,1}\left(z_{1}\right)$ and $\eta_{2,2}\left(z_{1}\right)$ gives $\eta_{2,1}\left(z_{1}\right)=\eta_{2,2}\left(z_{1}\right)=0$.

Recall that the nonzero order parameters $\eta_{\perp}(z), \eta_{\|}(z)$, and $\eta_{2,0}(z)$ are defined as

$$
\begin{aligned}
\eta_{\perp}(z) & =\langle\cos \theta\rangle, \\
\eta_{\|}(z) & =\langle\sin \theta \cos \varphi\rangle, \\
\eta_{2,0}(z) & =\left\langle P_{2}(\cos \theta)\right\rangle,
\end{aligned}
$$

where $P_{2}(\cos \theta)$ is the Legendre polynomial of the second order and $\langle\cdots\rangle$ denotes the orientation average weighted by $\hat{f}(z, \omega) . \quad \eta_{\perp}(z)$ is a component of the unit vector $\left\langle\vec{\mu}_{0}(\omega) / \mu_{0}\right\rangle$ in the $z$ direction and $\eta_{\|}(z)$ is the component in the $x$ direction. On the basis of Eqs. (18) and (19) it follows that if $E_{\perp}$ or $E_{\|}$is zero, either $\eta_{\perp}(z)$ or $\eta_{\|}(z)$ will be zero. If both $E_{\perp}$ and $E_{\|}$are zero (in the zero field), $\eta_{2,0}(z)$ will become the only nonzero order parameter.

\section{BULK-FLUID PROPERTIES}

\section{A. Slab-shaped system}

\section{Grand potential and order parameters}

For the bulk-fluid phases the density and order parameters are constant, denoted as $\rho, \eta_{\perp}, \eta_{\|}$, and $\eta_{20}$, respectively. With the condition $\int_{-\infty}^{+\infty} \phi_{2}(z) d z=0$, Eq. (20) gives that the order parameter $\eta_{2,0}=0$, and with the condition $\int_{-\infty}^{+\infty} \phi_{1}(z) d z=-(8 \pi / 3) \mu_{0}^{2}$, Eqs. (18) and (19) give that

$$
\eta_{\perp}=\frac{\beta \mu_{0}}{3}\left(E_{\perp}-\frac{8 \pi}{3} P_{\perp}\right) \text { and } \eta_{\|}=\frac{\beta \mu_{0}}{3}\left(E_{\|}+\frac{4 \pi}{3} P_{\|}\right),
$$


where $P_{\perp}$ and $P_{\|}$are the two components of the polarization vector in the bulk phases

$$
P_{\alpha}=\mu_{0} \eta_{\alpha} \rho \quad(\alpha=\perp, \|) .
$$

We introduce the vector

$$
\vec{K}=\vec{E}^{M}+\frac{4 \pi}{3} \vec{P}
$$

thereby Eq. (22) can be written in the more compact form

$$
\eta_{\alpha}=\beta \mu_{0} K_{\alpha} / 3 \quad(\alpha=\perp, \|) .
$$

In Eq. (24) $\vec{E}^{M}$ is the so-called Maxwell field [27], defined as the spatial-time averaging of microscopic fields of molecules. As a result, the Maxwell field is dependent on the geometry of the dielectric system. For the slab-shaped system the two components of $\vec{E}^{M}$ are given by $[28,29]$

$$
E_{\alpha}^{M}=E_{\alpha}-4 \pi P_{\alpha} \delta_{\alpha, \perp} \quad(\alpha=\perp, \|),
$$

where $E_{\alpha}$ is component of the applied external field and $\delta_{\alpha, \perp}$ is the Kronecker delta function. The term $4 \pi P_{\perp}$ describes the induced field resulting from the charge induced at the macroscopic surfaces of the slab. Introducing the dielectric constant $\varepsilon$ via the equation

$$
4 \pi \vec{P}=(\varepsilon-1) \vec{E}^{M},
$$

and combining it with Eqs. (23)-(25) give the ClausiusMossotti formula

$$
\varepsilon=\frac{1+2 y}{1-y},
$$

where $y=(4 \pi / 9) \beta \mu_{0}^{2} \rho$, a known result for weakly polar fluids based on the mean-field theory.

Equations (22) and (23) also give an expression for $\eta_{\perp}$ and $\eta_{\|}$as a function of the applied field, density, and temperature:

$$
\eta_{\perp}=\frac{\beta \mu_{0} E_{\perp}}{3(1+2 y)} \quad \text { and } \quad \eta_{\|}=\frac{\beta \mu_{0} E_{\|}}{3(1-y)}
$$

By using Eq. (29), the grand potential [Eq. (15)] and the chemical potential [Eq. (17)] can be written as

$$
\begin{gathered}
\frac{\Omega}{V}=f_{h s}(\rho)-\frac{1}{2} \Psi \rho^{2}-\mu \rho-\frac{1}{2} \mu_{0} \rho\left(\eta_{\perp} E_{\perp}+\eta_{\|} E_{\|}\right), \\
\mu=\mu_{h s}(\rho)-\Psi \rho-\frac{3}{2 \beta}\left(\eta_{\perp}^{2}+\eta_{\|}^{2}\right),
\end{gathered}
$$

where $V$ is the volume of the system and $\Psi$ $=\left(16 \pi \varepsilon d^{3} / 3\right)\left[1+(2 / 3) \beta \epsilon+(1 / 12)\left(\beta \mu_{0}^{4} / d^{6} \varepsilon\right)\right]$.

In general, the grand potential $\Omega$ of a dielectric system subjected to a uniform electric field is given by $[29,30]$

$$
\frac{\Omega(\mu, T)}{V}=\frac{\Omega_{0}(\mu, T)}{V}-\frac{1}{2}(\vec{P} \cdot \vec{E}),
$$

where $\vec{P}$ is the polarization per unit volume and $\Omega_{0}$ is the grand potential in the zero field but under the condition that the chemical potential $\mu$ is the same as that in the presence of the electric field. Equation (30) is consistent with Eq. (32), provided that $\Omega_{0} / V=f_{h s}(\rho)-(1 / 2) \Psi \rho^{2}-\mu \rho$ and Eq. (23). Moreover, the general expression for the chemical potential of the dielectric system subjected to the electric field is given by $[29,30]$

$$
\mu=g-\frac{\left(E^{M}\right)^{2}}{8 \pi} \frac{\partial \varepsilon}{\partial \rho}
$$

where $g$ is the chemical potential of the system in zero field. Again, one can show that Eq. (31) is consistent with Eq. (33), given that $g=\mu_{h s}(\rho)-\Psi \rho$ and that

$$
\frac{3}{2 \beta}\left(\eta_{\perp}^{2}+\eta_{\|}^{2}\right)=\frac{1}{6} \beta \mu_{0}^{2} K^{2}=\frac{\left(E^{M}\right)^{2}}{8 \pi} \frac{\partial \varepsilon}{\partial \rho} .
$$

Equation (34) can be derived from Eqs. (24), (27), and (28) which yields

$$
\vec{E}^{M}=\frac{3 y}{(\varepsilon-1)} \vec{K} \quad \text { and } \quad \frac{\partial \varepsilon}{\partial \rho}=\frac{(\varepsilon-1)^{2}}{3 y \rho} .
$$

Combining Eq. (35) with Eq. (24) yields Eq. (34).

\section{Vapor-liquid coexistence}

The simultaneous solution of the equations $\mu\left(\rho_{l}\right)$ $=\mu\left(\rho_{v}\right)$ and $\Omega\left(\rho_{l}\right)=\Omega\left(\rho_{v}\right)$ at a given $T$ results in the coexisting liquid $\rho_{l}$ and vapor $\rho_{v}$ densities. Hereafter, we will express the thermodynamic variables in dimensionless units, i.e., $\xi=\left(\pi d^{3} / 6\right) \rho, \quad T^{*}=1 / \beta \epsilon, \quad E^{*}=E\left(d^{3} / \epsilon\right)^{1 / 2}$, and $\mu_{0}^{*}$ $=\mu_{0} /\left(d^{3} \varepsilon\right)^{1 / 2}$. Denoting the angle between the direction of $\vec{E}$ and the surface normal of the slab as $\theta_{E}$, we thus have $E_{\perp}=E \cos \theta_{E}$ and $E_{\|}=E \sin \theta_{E}$. Because the MMF DFT [3] is applicable for weakly dipolar fluids $\left(\mu_{0}^{*} \leqslant 1\right)$ (Ref. [16]), here we consider the dipolar fluid with the reduced dipole moment $\mu_{0}^{*}=1$.

In Fig. 1 we plot the coexisting vapor-liquid densities in the cases of $E^{*}=0$ and $E^{*}=0.5$ with the angle $\theta_{E}$ $=0, \pi / 4, \pi / 3$, and $\pi / 2$. Figure 1 shows that when the electric field is perpendicular to the slab surfaces $\left(\theta_{E}=0\right)$ the critical temperature $T_{c}^{*}$ is lowered; at a fixed $T^{*}$ the coexisting vapor density $\xi_{v}$ is increased while the coexisting liquid density $\xi_{l}$ is decreased, compared to the results in the zero field. Increasing $\theta_{E}$ from 0 to $\pi / 2$ results in a higher $T_{c}^{*}$, a lower $\xi_{v}$, and a higher $\xi_{l}$. Therefore, as shown in Fig. 1, at a given $T^{*}$ the effect of increasing the field strength on the coexisting densities can be offset by a change of the field direction [31]. We also find that the difference between the coexisting liquid and vapor density near $T_{c}\left(E, \theta_{E}\right)$ satisfies the scaling relation

$$
\xi_{l}-\xi_{v} \sim \tau^{1 / 2},
$$



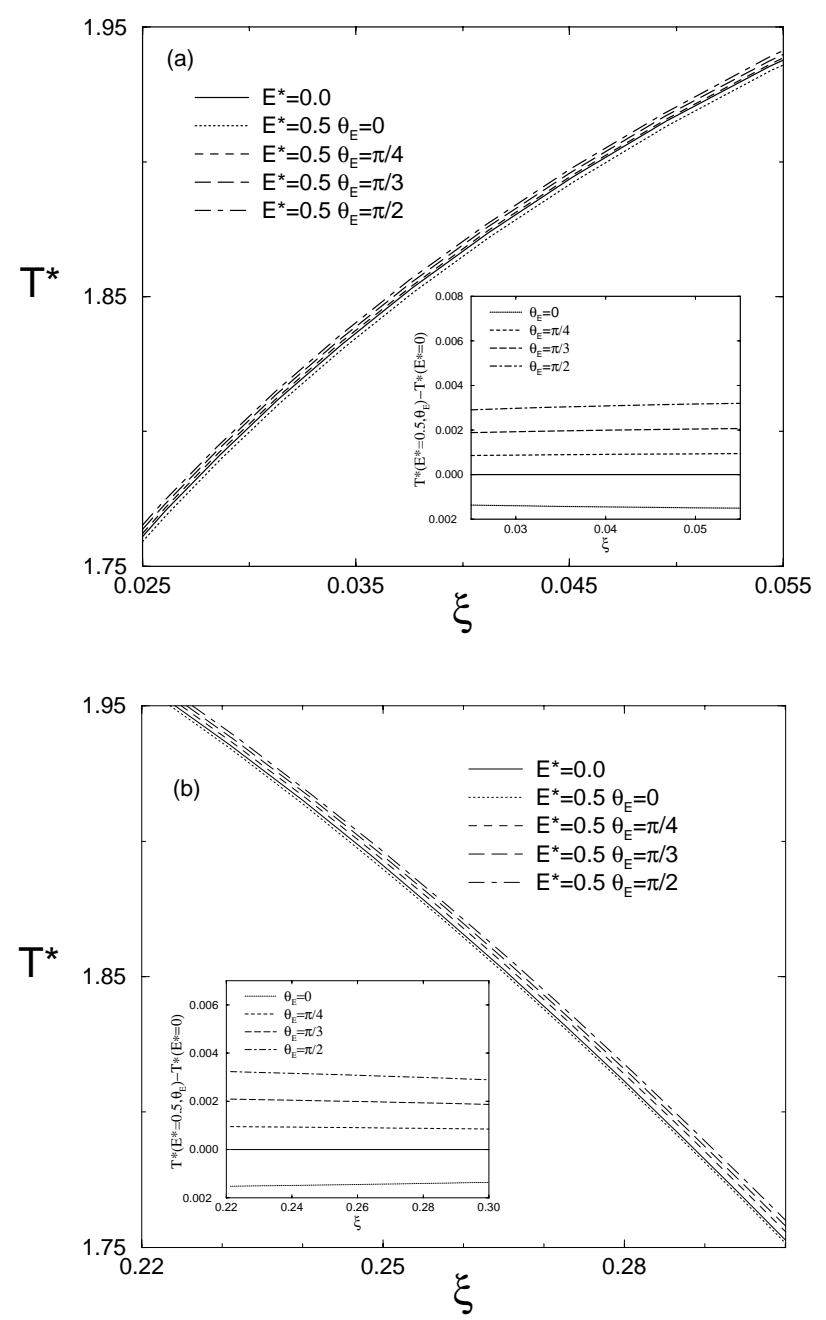

FIG. 1. Vapor-liquid coexistence curves for various given values of electric field and field directions. (a) The vapor branch and (b) the liquid branch. The inset shows the differences in the coexistence curves from the zero-field curve.

where $\tau \equiv 1-T^{*} / T_{c}^{*}$, regardless of the strength and direction of the field.

From Eq. (28), the temperature dependence of the dielectric constants $\varepsilon_{v}$ and $\varepsilon_{l}$ at the coexisting vapor and liquid densities can be calculated. The dielectric-constant curves exhibit similar behavior to the coexisting-density curves (Fig. 1): the field that is normal to the interface increases $\varepsilon_{v}$ and $\varepsilon_{l}$ whereas the field in parallel to the interface decreases $\varepsilon_{v}$ and $\varepsilon_{l}$. Near $T_{c}^{*}$ the difference $\varepsilon_{l}-\varepsilon_{v}$ satisfies the scaling relation as Eq. (36), i.e.,

$$
\varepsilon_{l}-\varepsilon_{v} \sim \tau^{1 / 2}
$$

In Table I, we list the critical temperature $T_{c}^{*}$, critical density $\xi_{c}$, and critical dielectric constant $\varepsilon_{c}$ for various given $E^{*}$ and $\theta_{E}$. One can see that when the field is normal to the slab surfaces $\left(E^{*}=0.5, \theta_{E}=0\right) T_{c}^{*}$ is slightly reduced whereas when the field is in parallel with the surfaces $\left(E^{*}\right.$ $\left.=0.5, \theta_{E}=\pi / 2\right) T_{c}^{*}$ is increased. The critical density $\xi_{c}$ is always increased by the field and the increase is nearly inde-
TABLE I. Values of the critical temperature $T_{c}^{*}$, density $\xi_{c}$, dielectric permittivity $\varepsilon_{c}$, and orientation order parameters $\eta_{\perp c}$ and $\eta_{\|_{c}}$ in the slab-shaped system for various given $E^{*}$ and $\theta_{E}$.

\begin{tabular}{ccccccc}
\hline \hline$E^{*}$ & $\theta_{E}$ & $T_{c}^{*}$ & $\xi_{c}$ & $\varepsilon_{c}$ & $\eta_{\perp c}$ & $\eta_{\| c}$ \\
\hline 0.0 & & 2.0590 & 0.1304 & 1.6096 & 0.0000 & 0.0000 \\
0.5 & 0 & 2.0574 & 0.1306 & 1.6113 & 0.0605 & 0.0000 \\
0.5 & $\pi / 4$ & 2.0600 & 0.1306 & 1.6104 & 0.0428 & 0.0688 \\
0.5 & $\pi / 3$ & 2.0612 & 0.1306 & 1.6099 & 0.0302 & 0.0843 \\
0.5 & $\pi / 2$ & 2.0624 & 0.1306 & 1.6095 & 0.0000 & 0.0972 \\
\hline \hline
\end{tabular}

pendent of the field direction. For the critical dielectric constant $\varepsilon_{c}$ the field effect is opposite to that for $T_{c}^{*}$, that is, changing the field direction from $\theta_{E}=0$ to $\pi / 2$ will increase $\varepsilon_{c}$, but reduce $T_{c}^{*}$.

In Figs. 2(a) and 2(b) we plot the phase coexistence
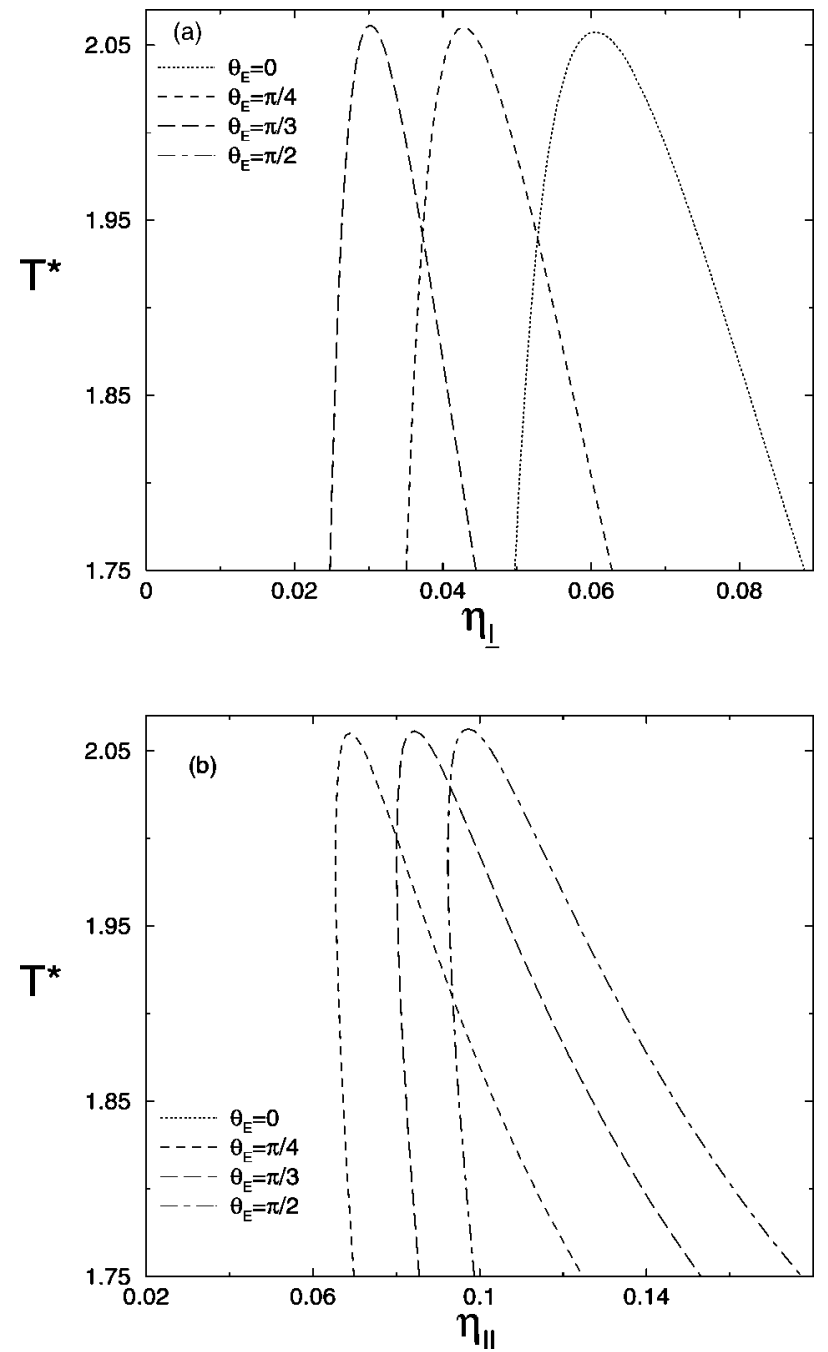

FIG. 2. The orientation order parameter (a) $\eta_{\perp}$, and (b) $\eta_{\|}$at the vapor-liquid coexistence for various given field directions. Note that $\eta_{\perp}=0$ for $\theta_{E}=\pi / 2$ and $\eta_{\|}=0$ for $\theta_{E}=0$. At a fixed $\theta_{E}$, (a) the branch with larger $\eta_{\perp}$ values corresponds to the vapor phase and (b) the branch with smaller $\eta_{\|}$values corresponds to the vapor phase. 
curves for the orientation order parameters $\eta_{\perp}$ and $\eta_{\|}$. For given $T^{*}, E^{*}$, and $\theta_{E}$ the branch of the curve having larger values of $\eta_{\perp}$ corresponds to the vapor phase whereas the branch having smaller values corresponds to the liquid phase [Eq. (29)]. In contrast, the branch having smaller values of $\eta_{\|}$corresponds to the vapor phase whereas the branch having larger values of $\eta_{\|}$corresponds to the liquid phase. Since a field that is normal to the surfaces tends to force the molecular dipoles aligning in the same direction, one can see from Fig. 2 that when $\eta_{\perp}$ is at the maximum, $\eta_{\|}$is zero. As $\theta_{E}$ increases from zero, the tendency for dipoles to be in the direction normal to the surfaces becomes weaker. As a result, $\eta_{\perp}$ becomes smaller while $\eta_{\|}$becomes larger. Similarly, since a field that is parallel to the surfaces $\left(\theta_{E}=\pi / 2\right)$ tends to force the dipoles to align in the same direction, when $\eta_{\|}$is at the maximum $\eta_{\perp}$ is zero. We also find that both $\eta_{\perp, v}$ $-\eta_{\perp, l}$ and $\eta_{\|, l}-\eta_{\|, v}$, where $\eta_{\alpha, v}$ and $\eta_{\alpha, l}(\alpha=\perp, \|)$ are the order parameters $\eta_{\perp}$ and $\eta_{\|}$at phase coexistence, follow the same scaling relation:

$$
\eta_{\perp, v}-\eta_{\perp, l} \sim \tau^{1 / 2} \quad \text { and } \quad \eta_{\|, l}-\eta_{\|, v} \sim \tau^{1 / 2},
$$

when $\tau \ll 1$. The critical values of $\eta_{\perp c}$ and $\eta_{\| c}$ are also listed in Table I. The effect of the field direction on $\eta_{\perp c}$ and $\eta_{\|_{c}}$ is more pronounced compared with that on $T_{c}^{*}, \xi_{c}$, and $\varepsilon_{c}$. Changing the field direction from $\theta_{E}=0$ to $\pi / 2$ reduces $\eta_{\perp c}$ but increases $\eta_{\|_{c}}$. We calculated the ratio of the maximum value of $\eta_{\| c}$ to the maximum value of $\eta_{\perp c}$, which is about 1.61. The ratio indicates that the effect due to the parallel field on the dipole ordering, near the critical point, is greater than that due to the normal field.

Some qualitative features of the vapor-liquid coexistence can be understood on the basis of the grand potential $\Omega$, which can be written in the van der Waals form via Eqs. (29)-(31),

$$
\Omega / V=-p_{h s}(\rho)+\frac{1}{2} \Psi_{e f f} \rho^{2},
$$

where $p_{h s}=\rho \mu_{h s}(\rho)-f_{h s}(\rho)$ is the hard-sphere pressure and $\Psi_{e f f}$ is a measure of the effective intermolecular attraction, i.e.,

$$
\Psi_{e f f}=\Psi+\frac{4 \pi}{3} \mu_{0}^{2}\left(\eta_{\|}^{2}-2 \eta_{\perp}^{2}\right)
$$

Since $\eta_{\|}=0$ in the normal field, $\Psi_{\text {eff }}$ is less than $\Psi$. When the field direction is changed from the normal direction, $\Psi_{\text {eff }}$ gradually increases until it arrives at the maximum in the parallel field where $\eta_{\perp}=0$. The increase of the effective intermolecular attraction gives rise to a higher $T_{c}^{*}$. This explains why $T_{c}^{*}$ increases with increasing angle $\theta_{E}$. The fact that a parallel field results in a larger shift of the coexistence curve than a normal field is due to the induced field $4 \pi P_{\perp}$, which arises only in the normal field. Because the induced field acts in the opposite direction to the applied field, it reduces the effective intermolecular attraction.

In Fig. 3 we plot all the coexistence curves using the scaled temperature $T^{*} / T_{c}^{*}\left(E, \theta_{E}\right)$, scaled density
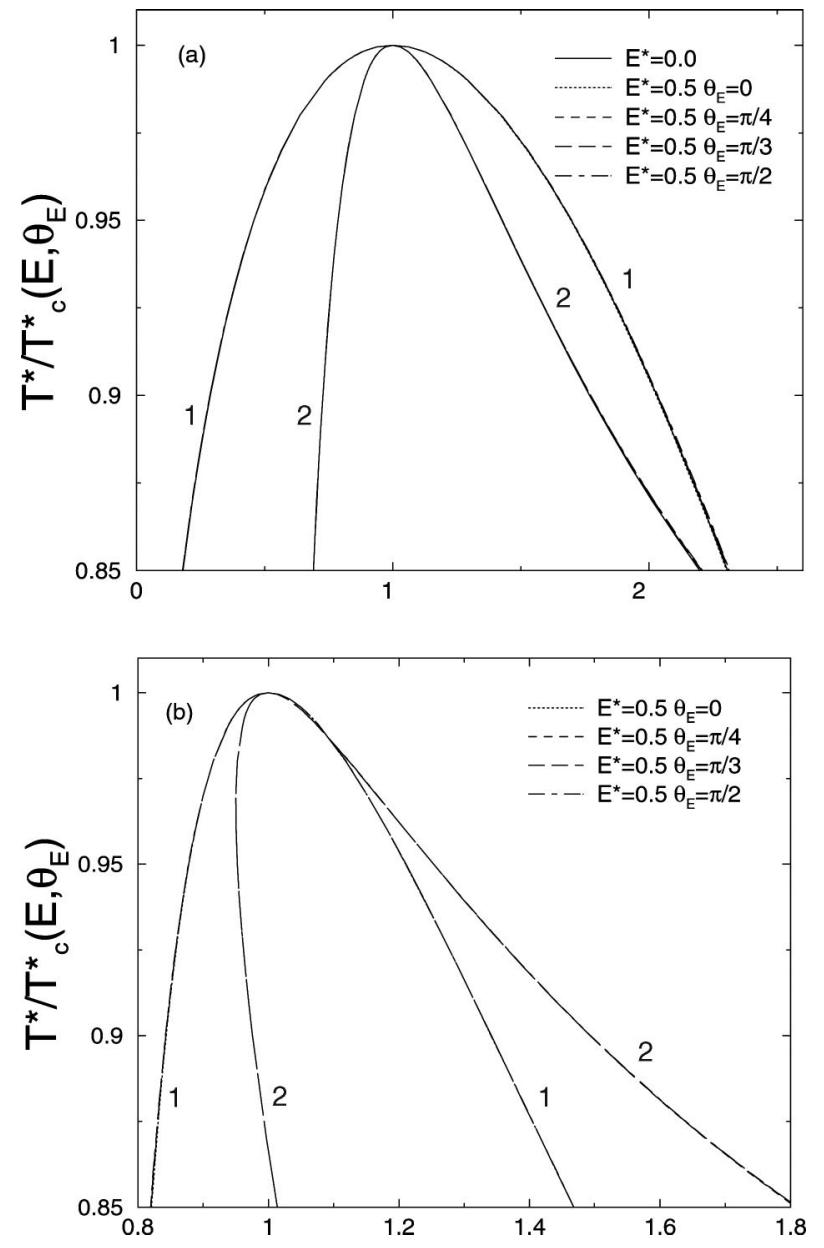

FIG. 3. (a) The scaled vapor-liquid coexistence curves for densities (curves 1) and dielectric constants (curves 2) for various given $\theta_{E}$. The temperatures, densities, and dielectric constants are divided by the corresponding critical value. The horizontal axis is $\xi / \xi_{c}\left(E, \theta_{E}\right)$ for curve 1 and $\varepsilon / \varepsilon_{c}\left(E, \theta_{E}\right)$ for curve 2. (b) The scaled vapor-liquid coexistence curves for order parameters $\eta_{\perp}$ (curves 1 ) and $\eta_{\|}$(curves 2) for various given $\theta_{E}$. The horizontal axis is $\eta_{\perp} / \eta_{\perp c}\left(E, \theta_{E}\right)$ for curves 1 and $\eta_{\|} / \eta_{\| c}\left(E, \theta_{E}\right)$ for curves 2 .

$\xi / \xi_{c}\left(E, \theta_{E}\right)$, scaled dielectric constant $\varepsilon / \varepsilon_{c}\left(E, \theta_{E}\right)$, and scaled order parameters $\eta_{\perp} / \eta_{\perp c}\left(E, \theta_{E}\right)$ and $\eta_{\|} / \eta_{\| c}\left(E, \theta_{E}\right)$. For given values of $E^{*}$ and $\theta_{E}$ we find that the density, dielectric constant, and order-parameter curves at the phase coexistence can all be collapsed onto a master curve. We therefore conclude that the laws of the corresponding states are satisfied for these quantities in nonzero fields.

\section{B. Sphere-shaped system}

\section{Grand potential and order parameters}

In this section we consider a sphere-shaped homogeneous dielectric system surrounded by a medium having the dielectric constant $\varepsilon^{\prime}$. Again, for a homogeneous system we have $\rho(z) \rightarrow \rho$ and $\hat{f}(z, \omega) \rightarrow \hat{f}(\omega)$, as described in Sec. I. Letting the $z$ axis be the direction of the electric field $\vec{E}$ and taking into account the symmetry of the system, the smaller anisotropic part of the orientation distribution function $\Delta \hat{f}(\omega)$ can 
be written as an expansion of the Legendre polynomial $P_{i}(\cos \theta)$, i.e., $\Delta \hat{f}(\omega)=\sum_{i=1}^{\infty}[(2 i+1) / 2] \eta_{i} P_{i}(\cos \theta)$, where $\theta$ is the angle between the dipole vector and the $z$ axis. The expansion coefficients $\left\{\eta_{i}\right\}$ are denoted as the order parameters.

First, we consider the expansion up to the third order. The grand potential per unit volume $\Omega / V$ of the system can be written as

$$
\begin{aligned}
\frac{\Omega}{V}= & f_{h s}(\rho)+\frac{\rho}{\beta}\left(\frac{3}{2} \eta_{1}^{2}+\frac{5}{2} \eta_{2}^{2}-3 \eta_{1}^{2} \eta_{2}-\frac{25}{21} \eta_{2}^{3}\right) \\
& -\frac{1}{2} \Psi \rho^{2}-\frac{2}{45} \frac{\pi}{d^{3}} \mu_{0}^{4} \eta_{2}^{2} \rho^{2}-\mu \rho-\mu_{0} \rho \eta_{1} E \\
& -\frac{4 \pi}{3} \frac{\left(\varepsilon^{\prime}-1\right)}{\left.2 \varepsilon^{\prime}+1\right)} \mu_{0}^{2} \eta_{1}^{2} \rho^{2} .
\end{aligned}
$$

The sum in parentheses results from the orientation entropy [Eq. (6)] with the ln-term expanded to the third order of $\Delta \hat{f}$. The next two terms in Eq. (41) result from the interaction part of free energy [Eq. (7)]. The last term in Eq. (41) has an $\varepsilon^{\prime}$ dependence, due to the reaction field induced by the surrounding media [33]. Minimizing $\Omega / V$ with respect to $\left\{\eta_{i}\right\}$ gives

$$
\begin{gathered}
\eta_{1}=\frac{\beta \mu_{0} E / 3}{1-(8 \pi / 9)\left[\left(\varepsilon^{\prime}-1\right) /\left(2 \varepsilon^{\prime}+1\right)\right] \mu_{0}^{2} \beta \rho-2 \eta_{2}}, \\
\eta_{2}=\frac{3 \eta_{1}^{2} / 5}{1-(4 / 225)\left(\beta \mu_{0}^{4} \pi / d^{3}\right) \rho-(5 / 7) \eta_{2}} .
\end{gathered}
$$

$\eta_{1}$ and $\eta_{2}$ in the above equations can be separated with the help of an iterative procedure. To the first-order (in $\eta_{1}$ ) approximation, $\eta_{1}$ and $\eta_{2}$ can be written as

$$
\eta_{1}=\frac{\beta \mu_{0} E}{\left.3\left\{1-\left[2\left(\varepsilon^{\prime}-1\right) / 2 \varepsilon^{\prime}+1\right)\right] y\right\}}, \quad \eta_{2}=o\left(\eta_{1}^{2}\right) \approx 0,
$$

because $\beta \mu_{0} E \ll 1$. Thus, under this simple approximation the order parameter $\eta_{1}$ is dependent linearly on $E$ whereas $\eta_{2}$ can be omitted. Next, the expression for $\eta_{1}$ can be rewritten as

$$
\eta_{1}=\frac{\beta \mu_{0}}{3}\left[E+\frac{8 \pi}{3} \frac{\left(\varepsilon^{\prime}-1\right)}{\left(2 \varepsilon^{\prime}+1\right)} P\right]
$$

where the polarization

$$
P=\mu_{0} \rho \eta_{1} .
$$

For a sphere with polarization $P$ the Maxwell field in the sphere $E^{M}$ is given by [34-36]

$$
E^{M}=E-\frac{4 \pi}{\left(2 \varepsilon^{\prime}+1\right)} P .
$$

Equations (44) and (46) together yield a formula for $\eta_{1}$ similar to Eq. (25) for $\eta_{\perp}$ and $\eta_{\|}$in the slab-shaped system, i.e.,

$$
\eta_{1}=\beta \mu_{0} K / 3
$$

where $K$ is given by Eq. (24). It is convenient to let the vectors $\vec{E}, \vec{P}, \vec{E}^{M}$, and $\vec{K}$ be all in the $z$ axis direction. The projection of these vectors onto the $z$ axis are denoted as $E$, $P, E^{M}$, and $K$, respectively.

Minimizing $\Omega / V$ with respect to $\rho$ and then taking into account Eq. (43) gives an expression for the chemical potential $\mu$

$$
\mu=\mu_{h s}(\rho)-\Psi \rho-\frac{3}{2 \beta} \eta_{1}^{2} .
$$

Now the Clausius-Mossotti formula Eq. (28) for the dielectric constant $\varepsilon$ can be derived again from Eqs. (24), (27), (43), and (45). We therefore conclude that with the meanfield approximation the Clausius-Mossotti formula [Eq. (28)] is independent of the shape of the system and the surrounding medium.

\section{Vapor-liquid coexistence}

We calculated the vapor-liquid coexisting densities for $E^{*}=0.5$ with three different surrounding media: $\varepsilon^{\prime}=1, \varepsilon^{\prime}$ $=\varepsilon$, and $\varepsilon^{\prime}=\infty$. The corresponding order parameter [Eq. (43)] becomes

$$
\begin{gathered}
\eta_{1}=\frac{\beta \mu_{0} E}{3} \quad\left(\varepsilon^{\prime}=1\right), \\
\eta_{1}=\frac{\beta \mu_{0} E(1+y)}{3(1-y)(1+2 y)} \quad\left(\varepsilon^{\prime}=\varepsilon\right), \\
\eta_{1}=\frac{\beta \mu_{0} E}{3(1-y)} \quad\left(\varepsilon^{\prime}=\infty\right),
\end{gathered}
$$

where the expression of $\eta_{1}$ for $\varepsilon^{\prime}=\varepsilon$ is obtained with using Eq. (28). It can be seen that for $\varepsilon^{\prime}=1, \eta_{1}$ is independent of the fluid density. For $\varepsilon^{\prime}=\infty$, the expression for $\eta_{1}$ is similar to that for $\eta_{\|}$if we set $E_{\|}=E$ [the second expression in Eq. (29) for the slab-shaped system]. We find that for $\varepsilon^{\prime}=\varepsilon$ and $\varepsilon^{\prime}=\infty$ the electric field raises $T_{c}^{*}$, whereas for $\varepsilon^{\prime}=1$ the electric field has no effect on $T_{c}^{*}$. The latter result can be understood based on the grand potential $\Omega$ which can be written again in the form of Eq. (39) [obtained from Eqs. (41), (43), and (48)], where now $\Psi_{\text {eff }}=\Psi+\left[8 \pi\left(\varepsilon^{\prime}\right.\right.$ $\left.-1) /\left(2 \varepsilon^{\prime}+1\right)\right] \mu_{0}^{2} \eta_{1}^{2}$. Thus, for $\epsilon^{\prime}=1$ the grand potential will show no field dependence. In Fig. 4, the coexistence curves for $\eta_{1}$ are displayed.

The fact that the electric field raises $T_{c}^{*}$ for $\varepsilon^{\prime}=\infty$ has been reported previously based on Monte Carlo simulations [37-39]. Kiyohara et al. [39] have shown that for $\varepsilon^{\prime}=\varepsilon$ the electric field broadens the vapor-liquid coexistence curves although the shift of the curves is not as much as that for $\varepsilon^{\prime}=\infty$. Our results are in agreement with their finding. For $\varepsilon^{\prime}=1$ Kiyohara et al. have reported that the electric field 


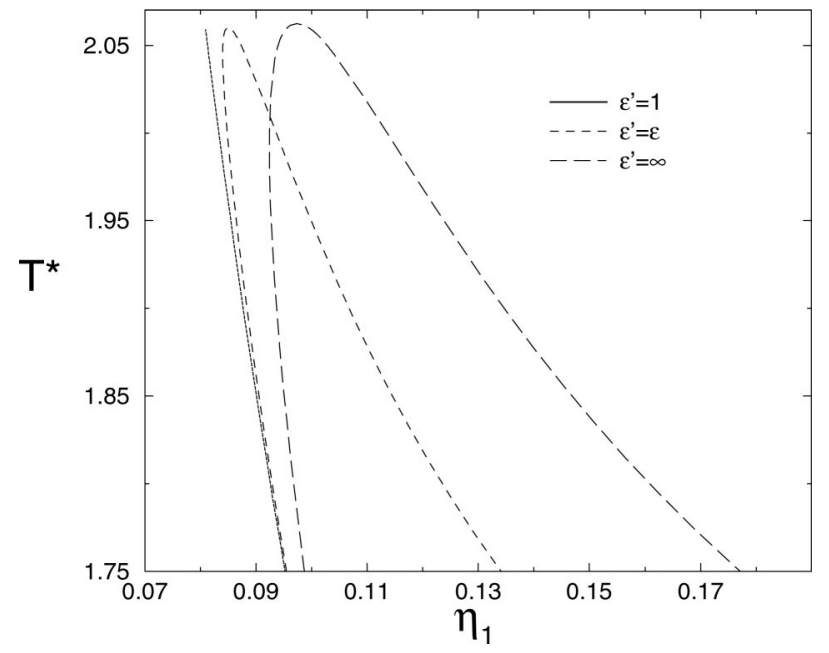

FIG. 4. The orientation order parameter $\eta_{1}$ at vapor-liquid coexistence for the sphere-shaped system with three different dielectric constants of the surrounding medium, $\varepsilon^{\prime}=1, \varepsilon$, and $\infty$.

slightly narrows the coexistence curve although the error bars in the density data appear to be larger than the total shift. Woodward and Nordholm [28] developed a different DFT to study the sphere-shaped dielectric system with $\varepsilon^{\prime}$ $=1$. They also found that the electric field narrows the coexistence curve. In their approach, the orientation correlation among dipoles were accounted for via the so-called CRAM potential, which renders their DFT beyond the mean-field approximation. We thus expect that if terms higher than $u_{\text {per }}^{2}$ in Eq. (7) were taken into account, a similar shift in the coexistence curve for $\varepsilon^{\prime}=1$ would also be seen.

Again, the coexisting densities also satisfy the law of corresponding states. This can be demonstrated by replotting the coexistence curves by using the axes $T^{*} / T_{c}^{*}\left(E, \varepsilon^{\prime}\right)$ and $\xi / \xi_{c}\left(E, \varepsilon^{\prime}\right)$. All curves are collapsed onto a single master curve. However, the order parameter $\eta_{1}$ at phase coexistence does not satisfy the law of corresponding states because the curves in Fig. 4 cannot be superimposed onto each other if the axes $T^{*} / T_{c}^{*}\left(E, \varepsilon^{\prime}\right)$ and $\eta_{1} / \eta_{1 c}\left(E, \varepsilon^{\prime}\right)$ were used.

Other results for the critical densities and temperatures in the electric field are summarized as follows: (i) The critical values for $E^{*}=0.5$ and $\varepsilon^{\prime}=1$ are nearly the same as those for $E^{*}=0$ except $\eta_{1 c}=0.0809$ (the first line in Table I); (ii) the critical values for $\varepsilon^{\prime}=\infty$ are nearly the same as those for the slab-shaped system when $\theta_{E}=\pi / 2$ and $\eta_{1 c}=\eta_{\| c}$ (the fifth line in Table I); (iii) the critical values for $\varepsilon^{\prime}=\varepsilon$ are $T_{c}^{*}=2.0601, \quad \xi_{c}=0.1292, \quad \varepsilon_{c}=1.6025$, and $\eta_{1 c}=0.0805$; (iv) near the critical temperature $T_{c}\left(E, \varepsilon^{\prime}\right)$ the coexisting densities (and dielectric constants) also follow the scaling relations Eqs. (36) and (37), respectively; (v) the difference in the order parameters at the phase coexistence for $\varepsilon^{\prime}=\varepsilon$ and $\varepsilon^{\prime}=\infty$ satisfy the scaling relations as Eq. (38), i.e., $\eta_{1, l}$ $-\eta_{1, v} \sim \tau^{1 / 2}$.

\section{INHOMOGENEOUS SLAB-SHAPED DIPOLAR SYSTEM}

In this section we consider interfacial properties of the slab-shaped system and their dependence on the applied electric field.
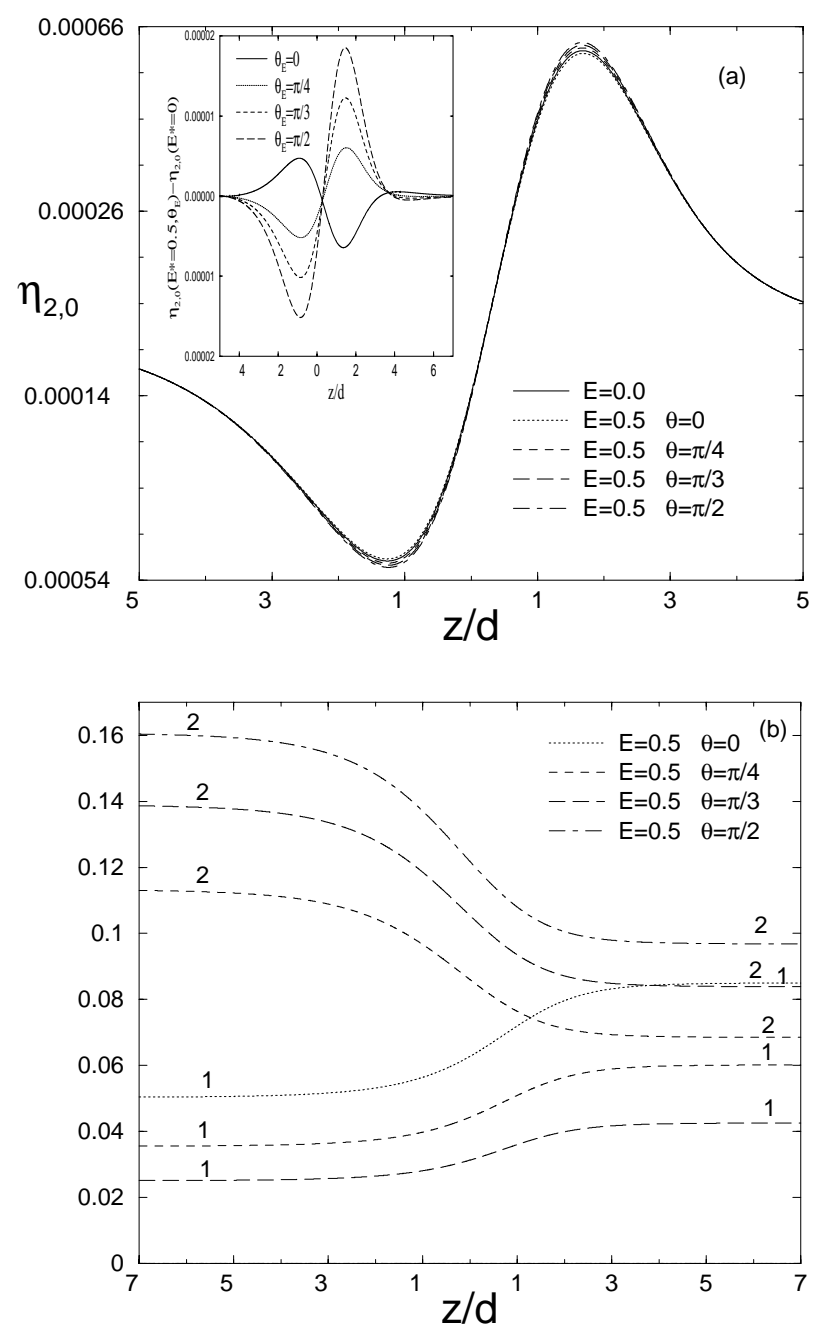

FIG. 5. The orientation order-parameter profiles (a) $\eta_{2,0}(z)$, and (b) $\eta_{\perp}(z)$ (curves 1) and $\eta_{\|}(z)$ (curves 2) for various given $\theta_{E}$ and $T^{*}=1.8$. The inset to (a) shows the differences in the $\eta_{2,0}(z)$ curves from the zero-field one.

\section{A. Density and orientation profiles}

We calculated the interfacial density profiles $\xi(z)$ and orientation profile $\eta_{\perp}(z), \eta_{\|}(z)$, and $\eta_{2,0}(z)$ by numerically solving the four equations (17)-(20) using an iteration method [1]. For given values of $E^{*}, \theta_{E}$, and $T^{*}$, the initial inputs for $\xi(z), \eta_{\perp}(z)$, and $\eta_{\|}(z)$ are taken as a step function with the bulk-liquid values $\xi_{l}, \eta_{\perp, l}$, and $\eta_{\|, l}$ set for $z$ $<0$ and the bulk-vapor values $\xi_{v}, \eta_{\perp, v}$, and $\eta_{\|, v}$ for $z$ $>0$. The initial input for $\eta_{2,0}(z)$ is just zero. Figures 5(a) and 5(b) show orientation profiles $\eta_{2,0}(z), \eta_{\perp}(z)$, and $\eta_{\|}(z)$ for $T^{*}=1.8$ and various given $E^{*}$ and $\theta_{E}$.

In the previous section, we showed that changing $\theta_{E}$ from 0 to $\pi / 2$ increases the liquid-side densities and decreases the vapor-side densities. Thus, it makes the density profile $\xi(z)$ steeper and reduces the 10-90 width of the interface $W$. Increasing the temperature $T^{*}$ toward $T_{c}^{*}$ leads to a divergence of $W$. Near $T_{c}^{*}\left(E, \theta_{E}\right)$ the mean-field scaling relation for $W$ is found to be

$$
W \sim \tau^{-1 / 2}
$$


for the given $E^{*}$ and $\theta_{E}$.

Figure 5(a) shows that $\eta_{2,0}(z)$ is weakly dependent on the field direction, whereas Fig. 5(b) shows that the orientation profiles $\eta_{\perp}(z)$ and $\eta_{\|}(z)$ are strongly sensitive to the field direction. In zero field, both $\eta_{\perp}(z)$ and $\eta_{\|}(z)$ become zero and $\eta_{2,0}(z)$ is the only nonzero order parameter; it describes the spontaneous interfacial ordering of dipoles. The spontaneous interfacial ordering of dipoles is well known from both experiments $[40,41]$ and theories $[3,7,14,16,42,43]$. Consistent with previous results, we find that the dipoles prefer to lie parallel to the interface on the liquid side $\left[\eta_{2,0}(z)<0\right]$, but perpendicular to the interface on the vapor side $\left[\eta_{2,0}(z)>0\right]$. As a measure of the degree of dipole orientational ordering at the interface we employed the $D$ parameter [16], that is, the difference between the maximal and minimal values of the order parameter $\eta_{2,0}(z)$, i.e., $D_{\eta_{2,0}}$ $\equiv \eta_{2,0}\left(z_{\max }\right)-\eta_{2,0}\left(z_{\min }\right)$, where $z_{\max }$ and $z_{\min }$ are the positions of the maximum and minimum on the $\eta_{2,0}(z)$ curve. The width of the profile $\eta_{2,0}(z)$ can be defined [16] by $W_{\eta_{2,0}} \equiv z_{\max }-z_{\text {min }}$. Figure 5(b) shows that when the electric field is perpendicular to the interface $\left(\theta_{E}=0\right)$ the $D_{\eta_{2,0}}$ parameter is slightly reduced but the width $W_{\eta_{2,0}}$ is increased. Changing the angle $\theta_{E}$ from 0 to $\pi / 2$ increases $D_{\eta_{2,0}}$ but reduces $W_{\eta_{2,0}}$.

Frodl and Dietrich [16] have shown that near $T_{c}^{*}$ the width $W_{\eta_{2,0}}$ and the $D_{\eta_{2,0}}$ parameter satisfy the scaling relations

$$
W_{\eta_{2,0}}=z_{\max }-z_{\min } \sim \tau^{-1 / 2} \quad \text { and } \quad D_{\eta_{2,0}} \sim \tau^{1 / 2}
$$

We find that both mean-field scaling relations still hold when the system is in the electric field.

Figure 5(b) shows that when the field is perpendicular to the interface $\left(\theta_{E}=0\right)$ the nonzero order parameter is $\eta_{\perp}(z)$ and $\eta_{\|}(z)=0$. Changing $\theta_{E}$ from 0 to $\pi / 2$ reduces the mean orientational ordering of the dipoles in the normal direction but enhances it in the direction parallel to the interface. For $\theta_{E}=\pi / 2, \eta_{\|}(z)$ becomes largest at every $z$ but $\eta_{\perp}(z)=0$. Both $\eta_{\perp}(z)$ and $\eta_{\|}(z)$ can be well approximated by an extended Eq. (29), i.e.,

$$
\eta_{\perp}(z)=\frac{\beta \mu_{0} E_{\perp}}{3[1+2 y(z)]} \quad \text { and } \quad \eta_{\|}(z)=\frac{\beta \mu_{0} E_{\|}}{3[1-y(z)]},
$$

where $y(z)=(4 \pi / 9) \beta \mu_{0}^{2} \rho(z)$.

More accurate formulas for $\eta_{\perp}(z)$ and $\eta_{\|}(z)$ than Eq. (52) can be obtained. To this end, we write $\phi_{1}\left(z_{12}\right)$ [appearing in Eqs. (18) and (19)] as

$$
\phi_{1}\left(z_{12}\right)=-\frac{8 \pi}{3} \mu_{0}^{2} \delta\left(z_{12}\right)+\phi_{1}\left(z_{12}\right)+\frac{8 \pi}{3} \mu_{0}^{2} \delta\left(z_{12}\right)
$$

with which Eq. (18) can be written as

$$
\begin{aligned}
\eta_{\perp}\left(z_{1}\right)= & \frac{\beta \mu_{0} E_{\perp}}{3}-\frac{8 \pi}{9} \beta \mu_{0}^{2} \rho\left(z_{1}\right) \eta_{\perp}\left(z_{1}\right) \\
& +\frac{8 \pi}{9} \beta \mu_{0}^{2} \int_{-\infty}^{\infty} d z_{2} \rho\left(z_{2}\right) \eta_{\perp}\left(z_{2}\right) \\
& \times\left[\frac{\phi_{1}\left(z_{12}\right)}{(8 \pi / 3) \mu_{0}^{2}}+\delta\left(z_{12}\right)\right]
\end{aligned}
$$

where $\delta\left(z_{12}\right)$ is the Dirac delta function. The two terms within the square brackets in Eq. (54) are negligibly small when $\left|z_{12}\right|>1.5$ for all temperatures considered. Within $\left|z_{12}\right|=1.5$, although $\xi\left(z_{2}\right)=\left(\pi d^{3} / 6\right) \rho\left(z_{2}\right)$ can vary by an order of magnitude, $\eta_{\perp}\left(z_{2}\right)$ varies only by $20 \%$ to $30 \%$. Thus, in the region $\left|z_{12}\right| \leqslant 1.5$ we can approximate $\eta_{\perp}\left(z_{2}\right)$ by $\eta_{\perp}\left(z_{1}\right)$ to arrive at an algebraic equation for $\eta_{\perp}\left(z_{1}\right)$. Solving this equation yields

$$
\eta_{\perp}\left(z_{1}\right)=\frac{\beta \mu_{0} E_{\perp}}{3\left[1+2 y\left(z_{1}\right)+I\left(z_{1}\right)\right]},
$$

where

$$
I\left(z_{1}\right)=-\frac{8 \pi}{9} \beta \mu_{0}^{2} \int_{-\infty}^{\infty} d z_{2} \rho\left(z_{2}\right)\left[\frac{\phi_{1}\left(z_{12}\right)}{(8 \pi / 3) \mu_{0}^{2}}+\delta\left(z_{12}\right)\right] .
$$

Similarly, using Eq. (53), Eq. (19) can be rewritten as

$$
\begin{aligned}
\eta_{\|}\left(z_{1}\right)= & \frac{\beta \mu_{0} E_{\|}}{3}+\frac{4 \pi}{9} \beta \mu_{0}^{2} \rho\left(z_{1}\right) \eta_{\|}\left(z_{1}\right) \\
& -\frac{4 \pi}{9} \beta \mu_{0}^{2} \int_{-\infty}^{\infty} d z_{2} \rho\left(z_{2}\right) \eta_{\|}\left(z_{2}\right) \\
& \times\left[\frac{\phi_{1}\left(z_{12}\right)}{(8 \pi / 3) \mu_{0}^{2}}+\delta\left(z_{12}\right)\right] .
\end{aligned}
$$

Replacing $\eta_{\|}\left(z_{2}\right)$ by $\eta_{\|}\left(z_{1}\right)$ and solving the resulting algebraic equation for $\eta_{\|}\left(z_{1}\right)$ yields

$$
\eta_{\|}\left(z_{1}\right)=\frac{\beta \mu_{0} E_{\|}}{3\left[1-y\left(z_{1}\right)-I\left(z_{1}\right) / 2\right]} .
$$

\section{B. Dielectric-permittivity profile}

At the interface, the dielectric permittivity is a tensor whose components can be determined from the formula

$$
\varepsilon_{\alpha}(z)=1+4 \pi \frac{P_{\alpha}(z)}{E_{\alpha}^{M}(z)} \quad(\alpha=\perp, \|),
$$

where

$$
P_{\alpha}(z)=\mu_{0} \rho(z) \eta_{\alpha}(z) \quad(\alpha=\perp, \|)
$$




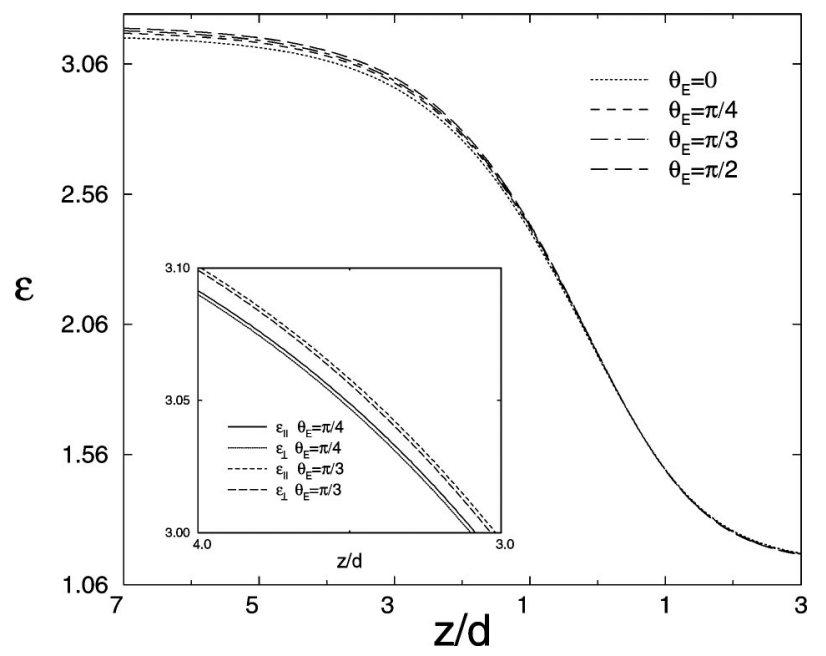

FIG. 6. The dielectric-permittivity profiles $\varepsilon(z)$ for various $\theta_{E}$, and $E^{*}=0.5$ and $T^{*}=1.8$. The inset shows a portion of the profiles using a larger axis scale.

is the component of the local polarization vector, and where $E_{\alpha}^{M}(z)$ is the component of the Maxwell field which has the same expression as Eq. (26) [44],

$$
E_{\alpha}^{M}(z)=E_{\alpha}-4 \pi P_{\alpha}(z) \delta_{\alpha, \perp} \quad(\alpha=\perp, \|) .
$$

Substituting Eqs. (60) and (61) into Eq. (59) gives

$$
\varepsilon_{\perp}(z)=\left[1-4 \pi \frac{P_{\perp}(z)}{E_{\perp}}\right]^{-1} \quad \text { and } \quad \varepsilon_{\|}(z)=1+4 \pi \frac{P_{\|}(z)}{E_{\|}} .
$$

In Fig. 6 we plot the dielectric-permittivity profiles $\varepsilon(z)$ for $E^{*}=0.5$ and various given $\theta_{E}$ and $T^{*}=1.8$. Because the anisotropy of the dielectric permittivity is small, the tensor character of the dielectric permittivity cannot be seen in Fig. 6. The inset of Fig. 6 shows a small portion of the profiles where the two components of the dielectric-permittivity tensor can be clearly distinguished. We find that at the interface the dielectric permittivity is well described by the local Clausius-Mossotti formula

$$
\varepsilon(z)=\frac{1+2 y(z)}{1-y(z)}
$$

A small correction due to inhomogeneity to the local Clausius-Mossotti formula of $\varepsilon(z)$ can be given analytically. Substituting Eqs. (55), (58), and (60) into Eq. (62) results in a more accurate equation for $\varepsilon_{\perp}(z)$ and $\varepsilon_{\|}(z)$, i.e.,

$$
\varepsilon_{\perp}(z)=\frac{1+2 y(z)+I(z)}{1-y(z)+I(z)}
$$

and

$$
\varepsilon_{\|}(z)=\frac{1+2 y(z)-I(z) / 2}{1-y(z)-I(z) / 2}
$$

Castle and Lekner $[9,10]$ employed a different approach to investigate the anisotropy of the dielectric-permittivity profile at the vapor-liquid interface for a nonpolar but polarizable fluid. They obtained the same formulas for $\varepsilon_{\perp}(z)$ and $\varepsilon_{\|}(z)$ [Eqs. (64) and (65)] but a different formula for $I(z)$ [Eq. (56)]. They found that if a unit correlation function is assumed, then $I(\bar{z})=0$, and the dielectric-permittivity profile can be described by the local Clausius-Mossotti formula. They therefore concluded that for nonpolar fluids the dielectric anisotropy at the interface can be seen only if the correlation function differs from unity. Our calculation indicates that their conclusion cannot be generalized to dipolar fluids. This is because, even if the correlation function is assumed to be unity, $\phi_{1}(\bar{z}) /\left(8 \pi \mu_{0}^{2} / 3\right)$ still differs from $\delta(\bar{z})$, as follows from the formula (A15b) in Ref. [3]. Thus, $I(\bar{z}) \neq 0$, and Eqs. (64) and (65) cannot be reduced to Eq. (63).

Knowing the anisotropy of the dielectric function at the vapor-liquid interface is important to ellipsometry measurements of liquid interfacial structure, for instance, the determination of the interface thickness [6]. In ellipsometry experiments, the coefficient of ellipticity $\bar{\rho}=\left.\operatorname{Im}\left(r_{p} / r_{s}\right)\right|_{\theta_{B}}$ is measured; this is the imaginary component of the ratio of the complex reflection amplitudes at the Brewster angle $\theta_{B}\left(r_{p}\right.$ and $r_{s}$ are the reflection amplitudes for $p$ and $s$ polarizations, respectively). The real part of this ratio at the Brewster angle, $\left.\operatorname{Re}\left(r_{p} / r_{s}\right)\right|_{\theta_{B}}=0$. For light (with the optical wavelength $\lambda$ ) incident from a liquid onto a planar surface, the coefficient of ellipticity $\bar{\rho}$ can be expressed, to the first order in interfacial thickness $W / \lambda \ll 1$, by the equation [5]

$$
\bar{\rho}=\frac{\pi}{\lambda} \frac{\sqrt{\varepsilon_{l}+\varepsilon_{v}}}{\varepsilon_{l}-\varepsilon_{v}} \chi_{0}
$$

where

$$
\chi_{0}=\int_{-\infty}^{+\infty} d z\left\{\varepsilon(z)+\frac{\varepsilon_{l} \varepsilon_{v}}{\varepsilon(z)}-\varepsilon_{l}-\varepsilon_{v}\right\}
$$

When dielectric anisotropy is considered, $\bar{\rho}$ is still given by Eq. (66), but $\chi_{0}$ is replaced by $[9,45,46]$

$$
\chi=\int_{-\infty}^{+\infty} d z\left\{\varepsilon_{\|}(z)+\frac{\varepsilon_{l} \varepsilon_{v}}{\varepsilon_{\perp}(z)}-\varepsilon_{l}-\varepsilon_{v}\right\}
$$

To illustrate the extent to which the anisotropy of the dielectric function affects $\bar{\rho}$, we calculated $\chi / \chi_{0}$ for $T^{*}=1.8$ and $\theta_{E}=\pi / 4$ or $\pi / 3$, and found $\chi / \chi_{0}=0.97$. Thus, when the anisotropy of the interface is taken into account, the righthand side of Eq. (66) will be a few percent smaller.

\section{Surface tension}

The thermodynamic surface tension $\sigma$ is defined as the excess grand potential per unit area,

$$
\sigma=\left(\Omega-\Omega^{l, v}\right) / A,
$$




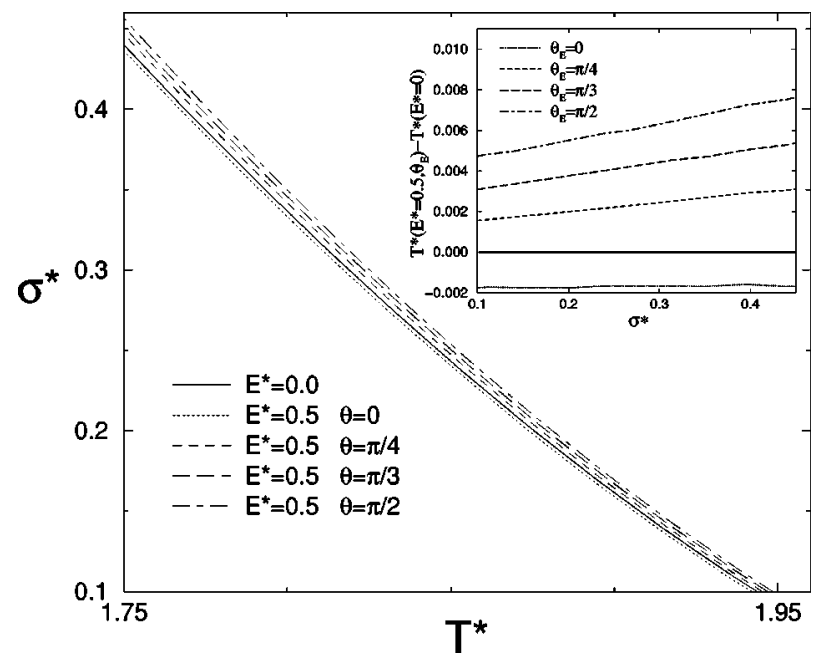

FIG. 7. Temperature dependence of the thermodynamic surface tension $\sigma^{*}=\sigma d^{2} / \varepsilon$ for various given $\theta_{E}$. The inset shows the differences in the temperature-tension curves from the zero-field curve.

where $\Omega^{l, v}$ is the grand potential at phase coexistence and $A$ is the surface area. Figure 7 shows the temperature dependence of $\sigma^{*}\left(=\sigma d^{2} / \varepsilon\right)$ for various given $\theta_{E}$. It can be seen that when the electric field is normal to the interface $\left(\theta_{E}\right.$ $=0)$ the field reduces the surface tension $\sigma^{*}$. Changing the angle $\theta_{E}$ from 0 to $\pi / 2$ increases the surface tension and $\sigma^{*}$ reaches a maximum for $\theta_{E}=\pi / 2$. We also find that the law of corresponding states is not applicable to $\sigma^{*}$; when we plot $\sigma^{*}$ versus the scaled temperature $\tau=1$ $-T^{*} / T_{c}^{*}\left(E, \theta_{E}\right)$, the $\sigma^{*}$ curves cannot be superimposed onto one another (except very close to the critical point). However, we find that the scaling relation [22]

$$
\sigma^{*} \sim \tau^{3 / 2}
$$

still holds for nonzero fields.

Another surface tension commonly shown in the literature is the mechanical surface tension $\gamma$, which is defined as onehalf of the trace of the excess surface tension tensor $\hat{\gamma}$, i.e.,

$$
\begin{gathered}
\gamma=\left(\gamma_{x}+\gamma_{y}\right) / 2, \\
A \gamma_{i}=-\int\left[p_{i i}(\vec{r})-p_{i i}^{l, v}(\vec{r})\right] d V \quad(i=x, y),
\end{gathered}
$$

where $p_{i i}(\vec{r})$ and $p_{i i}^{l, v}(\vec{r})$ are, respectively, a component of the local pressure tensor in the real interfacial system and in a fictitious system in which the liquid-side phase and vaporside phase are considered to be uniform up to a selected dividing surface. Assuming that the planar interface is in the $x-y$ plane and the electric field is in the $x-z$ plane, the two terms of $\gamma$ can be given by [31]

$$
\gamma_{x}=\sigma+\bar{P}_{\perp} E_{\perp} \quad \text { and } \quad \gamma_{y}=\sigma+\bar{P}_{\perp} E_{\perp}-\bar{P}_{\|} E_{\|}
$$

where $\bar{P}_{\perp}$ and $\bar{P}_{\|}$are the components of the excess surface polarization vector, and

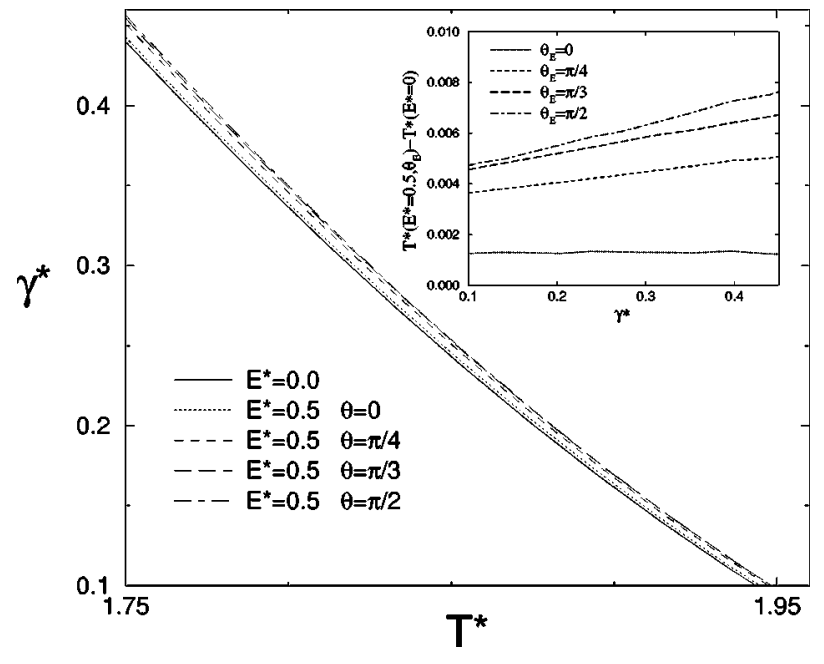

FIG. 8. Temperature dependence of scaled mechanical surface tension $\gamma^{*}=\gamma d^{2} / \varepsilon$ for various given $\theta_{E}$. The inset shows the differences in the temperature-tension curves from the zero-field curve.

$$
A \bar{P}_{\alpha}=\int\left[P_{\alpha}(\vec{r})-P_{\alpha}^{l, v}(\vec{r})\right] d V \quad(\alpha=\perp, \|),
$$

where $\vec{P}^{l, v}(\vec{r})$ is the polarization vector in the fictitious system.

In Fig. 8 we plot the temperature dependence of $\gamma^{*}$ $\left(\equiv \gamma d^{2} / \varepsilon\right)$ at the Gibbs equimolar dividing surface $Z_{G}$ for various given $\theta_{E}$. The Gibbs dividing surface is defined using the relation

$$
\int_{-\infty}^{Z_{G}}\left[\rho(z)-\rho_{l}\right] d z+\int_{Z_{G}}^{+\infty}\left[\rho(z)-\rho_{v}\right] d z=0 .
$$

In contrast to $\sigma^{*}, \gamma^{*}$ is always enhanced by the field regardless of the field direction. The enhancement is a minimum for $\theta_{E}=0$ and a maximum for $\theta_{E}=\pi / 2$. Like $\sigma^{*}$, the $\gamma^{*}-\tau$ relation does not satisfy the law of corresponding states, but it does follow the scaling relation

$$
\gamma^{*} \sim \tau^{3 / 2}
$$

Experimental studies of the field effect on the surface tension are scarce. Hurd and co-workers [32] found that the surface tension of a salt solution was reduced by an applied electric field $\left(10^{6} \mathrm{~V} / \mathrm{m}\right)$ perpendicular to the liquid surface. However, that result was questioned by Damm [47], who pointed out the sensitivity of their results to the conditions of the experiment. Hayes [48] measured the surface tension of pure water and a salt solution. He found no change in the surface tension with the field having a magnitude up to $10^{6} \mathrm{~V} / \mathrm{m}$.

Here, we have considered the dimensionless electric field $E^{*}=0.5$. To give an estimation of its physical magnitude, we utilize the Stockmayer parameters for methanol molecules [49], namely, $\mu_{0}^{*}=1.036, d=3.803 \AA$, and $\varepsilon / k_{B}$ $=359.0 \mathrm{~K}$ (the Stockmayer model is very similar to the 
dipolar model considered here). The calculation gives $E$ $\sim 10^{8} \mathrm{~V} / \mathrm{m}$. Therefore, to detect the field effect on the surface tension, a much stronger electric field than previously applied may be needed. It is expected that if the electric field is in parallel with the interface it will result in a greater change in the surface tension. However, care must be taken about the strength of the applied field, because if the electric field is above $5 \times 10^{8} \mathrm{~V} / \mathrm{m}$ it will lead to dielectric breakdown for most simple dielectric liquids [50].

\section{CONCLUSIONS}

We have studied the bulk and interfacial properties of a weakly dipolar fluid in an electric field. To this end, we have extended the MMF DFT of Teixeira and Telo da Gama to take into account the field effect. Particular attention has been paid to the effect of an electric field with an arbitrary direction with respective to the vapor-liquid interface.

For the bulk properties of a fluid in a slab-shaped system, we find that, when the electric field is perpendicular to the slab surfaces, it reduces the critical temperature $T_{c}^{*}$ and narrows the vapor-liquid coexistence curve, compared to zerofield results. Changing the direction of the field (by changing the angle $\theta_{E}$ between the field direction and the surface normal from 0 to $\pi / 2$ ) will increase $T_{c}^{*}$ and broaden the coexistence curve. When the electric field is parallel to the slab surfaces $\left(\theta_{E}=\pi / 2\right), T_{c}^{*}$ is higher than that in zero field. Near $T_{c}^{*}$, the differences in the density, dielectric constant, and order parameter at phase coexistence all follow the same scaling relation: $\xi_{l}-\xi_{v}, \varepsilon_{l}-\varepsilon_{v}, \quad \eta_{\perp, v}-\eta_{\perp, l}, \quad \eta_{\|, l}-\eta_{\|, v}$ $\sim \tau^{1 / 2}$.

We also investigated the properties of a sphere-shaped dielectric system in an electric field. In particular, we studied the dependence of vapor-liquid coexistence on the dielectric constant of the surrounding medium, $\varepsilon^{\prime}$. We find that for $\varepsilon^{\prime}=1$ the field has no effect on the coexistence curve. For $\varepsilon^{\prime}=\varepsilon$ and $\varepsilon^{\prime}=\infty$ the field increases $T_{c}^{*}$ and broadens the coexistence curve. The broadening is greater for $\varepsilon^{\prime}=\infty$. Our results are in good agreement with a computer simulation and previous theoretical studies in the cases of $\varepsilon^{\prime}=\varepsilon$ [39] and $\varepsilon^{\prime}=\infty$ [37-39]. However, we predict different behavior of the vapor-liquid coexistence in the case of $\varepsilon^{\prime}=1[28,39]$. We expect that higher terms in the expansion of the exponential in Eq. (7) should be included in order to see the field effect on the coexistence curve in the case of $\varepsilon^{\prime}=1$. Note that Groh and Dietrich [51] studied vapor-liquid coexistence in other non-slab-shaped systems, for example, a needleshaped spheroidal system. They also found that the critical temperature increases with increasing applied external field.

For the planar interfacial properties, we find that when the field is perpendicular to the interface $\left(\theta_{E}=0\right)$ the 10-90 interfacial width $W$ is broadened and the density profile $\xi(z)$ becomes smoother. Changing the field direction $\left(\theta_{E}\right)$ will decrease $W$ and make the slope of $\xi(z)$ at the interface steeper. The interfacial dipolar ordering can be described by the interfacial order-parameter profiles $\eta_{\perp}(z), \eta_{\|}(z)$, and $\eta_{2,0}(z)$ (the last is the only nonzero order parameter in the zero field). Using the parameter $D_{\eta_{2,0}}=\max \left(\eta_{2,0}\right)$ $-\min \left(\eta_{2,0}\right)$ as a measure of interfacial dipolar ordering, we find that for $\theta_{E}=0$ the field reduces $D_{\eta_{2,0}}$ and increases the width $W_{\eta_{2,0}}$ of the $\eta_{2,0}(z)$ profile. Increasing the angle $\theta_{E}$ from 0 to $\pi / 2$ will increase the $D_{\eta_{2,0}}$ parameter but decrease the width $W_{\eta_{2,0}}$. Near the critical point both $W$ and $W_{\eta_{2,0}}$ scale with the reduced temperature as $\tau^{-1 / 2}$, and $D_{\eta_{2,0}}$ as $\tau^{3 / 2}$. We also find that the dielectric-permittivity profile can be well approximated by the Clausius-Mossotti formula with a small correction due to the interfacial inhomogeneity.

Finally, we find that when the field is normal to the interface $\left(\theta_{E}=0\right)$ the thermodynamic surface tension $\sigma^{*}$ is reduced, compared to $\sigma^{*}$ in zero field. Increasing the angle $\theta_{E}$ from zero will enhance the surface tension $\sigma^{*}$, and $\sigma^{*}$ will reach a maximum for $\theta_{E}=\pi / 2$. In contrast, the mechanical surface tension $\gamma^{*}$ at the equimolar dividing surface is always increased by the field regardless of the field direction. The shift in $\gamma^{*}$ is minimal for $\theta_{E}=0$ and maximal for $\theta_{E}$ $=\pi / 2$. Both $\sigma^{*}$ and $\gamma^{*}$ satisfy the scaling $\sigma^{*}, \gamma^{*} \sim \tau^{3 / 2}$. Knowledge of the dependence of $\gamma^{*}$ on the direction of the electric field can be useful to the study of vapor-to-liquid nucleation in an electric field [52,53]. Thus far, experiments have not revealed any evidence of surface tension change due to an external electric field. We speculate that an electric field as high as $10^{8} \mathrm{~V} / \mathrm{m}$ may be needed in order to observe a notable field effect on the surface tension, and that the effect will become more pronounced when the electric field is applied along the interface.

\section{ACKNOWLEDGMENTS}

We are grateful to Professor A. Shchekin and Dr. T. Bykov for useful discussions. This work is supported by the National Science Foundation and in part by the Research Computing Facility of the University of Nebraska-Lincoln.

\section{APPENDIX}

Here we give the expression for the effective potential $\phi_{\text {eff }}$ appearing in Eq. (10). To obtain $\phi_{\text {eff }}\left(z, \omega_{1}, \omega_{2}\right)$, Ref. [3] used the condition of the invariance of the system in the $x-y$ plane. Therefore, the orientation distribution function depends only on the azimuthal angle $\theta$, namely, $f=f(z, \theta)$. Note that the average over the angle for those terms in the distribution function containing the factor $\cos \left(\varphi_{1}-\varphi_{2}\right)$ is zero. Thus, those terms are omitted in the expression of $\phi_{\text {eff }}\left(z, \omega_{1}, \omega_{2}\right)$ [Eq. (A13) in Ref. [3]].

For the system considered here, however, the invariant of the system in the $x-y$ plane no longer holds. As a result, the average over the angles for those terms containing $\cos \left(\varphi_{1}\right.$ $\left.-\varphi_{2}\right)$ in the distribution function $f(z, \omega)$ is no longer equal to zero because $f(z, \omega)$ has an axial angle $\varphi$ dependence. The final expression for the effective potential $\phi_{\text {eff }}$ in terms of spherical harmonics, accounting for its dependence on the axial angles $\varphi_{1}$ and $\varphi_{2}$, is given by 


$$
\begin{aligned}
\phi_{e f f}\left(z, \omega_{1}, \omega_{2}\right)= & A(z)+B(z) \frac{4 \pi}{3}\left[Y_{10}^{\prime} Y_{10}^{\prime \prime}+\frac{1}{2} Y_{1-1}^{\prime} Y_{11}^{\prime \prime}+\frac{1}{2} Y_{11}^{\prime} Y_{1-1}^{\prime \prime}\right]-\frac{\beta}{2}\left\{C(z)+D(z)\left[\frac{16 \pi}{45} Y_{20}^{\prime} Y_{20}^{\prime \prime}+\frac{8}{9} \sqrt{\frac{4 \pi}{5}}\left(Y_{20}^{\prime}+Y_{20}^{\prime \prime}\right)\right.\right. \\
& \left.+\frac{16}{9}\right]+E(z)\left[\frac{32 \pi}{5} Y_{20}^{\prime} Y_{20}^{\prime \prime}-\frac{8}{3} \sqrt{\frac{4 \pi}{5}}\left(Y_{20}^{\prime}+Y_{20}^{\prime \prime}\right)+\frac{16}{3}\right]+F(z)\left[\frac{32 \pi}{15} Y_{20}^{\prime} Y_{20}^{\prime \prime}+\frac{1}{3} \sqrt{\frac{4 \pi}{5}}\left(Y_{20}^{\prime}+Y_{20}^{\prime \prime}\right)\right] \\
& \left.+G(z) \frac{4 \pi}{5} Y_{20}^{\prime} Y_{20}^{\prime \prime}+H(z) \frac{4 \pi}{3}\left[Y_{10}^{\prime} Y_{10}^{\prime \prime}+\frac{1}{2} Y_{1-1}^{\prime} Y_{11}^{\prime \prime}+\frac{1}{2} Y_{11}^{\prime} Y_{1-1}^{\prime \prime}\right]\right\},
\end{aligned}
$$

where $Y_{l m}^{\prime}=Y_{l m}\left(\theta_{1}, \varphi_{1}\right), Y_{l m}^{\prime \prime}=Y_{l m}\left(\theta_{2}, \varphi_{2}\right)$, and the coefficients $A(z), B(z), C(z), D(z), E(z), F(z), G(z)$, and $H(z)$ are identical to those in Eq. (A14) of Ref. [3].

[1] V.B. Warshavsky, T.V. Bykov, and X.C. Zeng, J. Chem. Phys. 114, 504 (2001).

[2] V.B. Warshavsky and X.C. Zeng, Phys. Rev. E 68, 011203 (2003).

[3] P.I. Teixeira and M.M. Telo da Gama, J. Phys.: Condens. Matter 3, 111 (1991).

[4] P.I. Teixeira, B.S. Almeida, M.M. Telo da Gama, J.A. Rueda, and R.G. Rubio, J. Phys. Chem. 96, 8488 (1992).

[5] P. Drude, The Theory of Optics (Dover, New York, 1959), pp. 287-295.

[6] D. Beaglehole, Physica B 100, 163 (1980).

[7] E. Chacón, P. Tarazona, and G. Navascues, J. Chem. Phys. 79, 4426 (1983).

[8] P. Tarazona and G. Navascues, Mol. Phys. 47, 1021 (1982).

[9] P.J. Castle and J. Lekner, Physica A 101, 99 (1980).

[10] J. Lekner, Mol. Phys. 49, 1385 (1983).

[11] L. Liggeri, A. Sanfeld, and A. Steinchen, Physica A 206, 299 (1994)

[12] A.I. Rusanov and E.N. Brodskaya, J. Colloid Interface Sci. 62, 542 (1977).

[13] A.I. Rusanov and V.L. Kuz'min, Colloid J. USSR 39, 338 (1977).

[14] J. Eggebrecht, K.E. Gubbins, and S.M. Thompson, J. Chem. Phys. 86, 2286 (1987).

[15] S. Iatzevich and F. Forstmann, Mol. Phys. 98, 1309 (2000).

[16] P. Frodl and S. Dietrich, Phys. Rev. A 45, 7330 (1992).

[17] V. Talanquer and D.W. Oxtoby, J. Chem. Phys. 99, 4670 (1993).

[18] T.V. Bykov and X.C. Zeng, J. Phys. Chem. B 105, 11586 (2001).

[19] B. Yang, D.E. Sullivan, B. Tjipto-Margo, and C.G. Grey, Mol. Phys. 76, 709 (1992).

[20] V.B. Warshavsky and X.C. Zeng, J. Chem. Phys. 117, 3982 (2002).

[21] V.B. Warshavsky and X.C. Zeng, Phys. Rev. Lett. 89, 246104 (2002).

[22] P. Frodl and S. Dietrich, Phys. Rev. E 48, 3741 (1993).

[23] P. Frodl, B. Groh, and S. Dietrich, Ber. Bunsenges. Phys. Chem. 98, 503 (1994).

[24] T.M. Reed and K.E. Gubbins, Applied Statistical Mechanics (McGraw-Hill, New York, 1973), p. 117.

[25] J.O. Hirschfelder, C.F. Curtis, and R.B. Bird, Molecular Theory of Gases and Liquids (Wiley, New York, 1954), pp. 549-551.
[26] N.E. Carnahan and K.E. Starling, J. Chem. Phys. 51, 635 (1969).

[27] C.J.F. Böttcher, Theory of Electric Polarization (Elsevier, Amsterdam, 1973), Vol. 1.

[28] C.E. Woodward and S. Nordholm, J. Phys. Chem. 92, 501 (1988).

[29] J.S. Høye and G. Stell, J. Chem. Phys. 72, 1597 (1980).

[30] L.D. Landau and E.M. Lifshitz, Electrodynamics of Continuous Media (Pergamon, Oxford, 1984).

[31] E.N. Brodskaya and A.I. Rusanov, Colloid J. USSR 45, 558 (1983).

[32] C.M. Schmid, R.M. Hurd, and E.S. Snavely, Jr., Science 135, 791 (1961); J. Electrochem. Soc. 109, 852 (1962).

[33] B. Groh and S. Dietrich, Phys. Rev. E 50, 3814 (1994).

[34] H.G. Petersen, S.W. de Leeuw, and J.W. Perram, Mol. Phys. 66, 637 (1989).

[35] P.G. Kusalik, Mol. Phys. 81, 199 (1994).

[36] S.W. de Leeuw, J.W. Perram, and E.R. Smith, Proc. R. Soc. London, Ser. A 373, 27 (1980).

[37] M.J. Stevens and G.S. Grest, Phys. Rev. E 51, 5976 (1995).

[38] D. Boda, J. Winkelmann, J. Liszi, and I. Szalai, Mol. Phys. 87, 601 (1996).

[39] K. Kiyohara, K.J. Oh, X.C. Zeng, and K. Ohta, Mol. Simul. 23, 95 (1999).

[40] R. Superfine, J.Y. Huang, and Y.R. Shen, Phys. Rev. Lett. 66, 1066 (1991).

[41] M.C. Goh, J.M. Hicks, K. Kemnitz, G.R. Pinto, K. Bhattacharyya, K.B. Eisental, and T.F. Heinz, J. Phys. Chem. 96, 8488 (1992).

[42] F.M. Kuni and A.I. Rusanov, Russ. J. Phys. Chem. 43, 700 (1969).

[43] M. Kasch and F. Forstmann, J. Chem. Phys. 99, 3037 (1993).

[44] V.L. Kuz'min, Colloid J. USSR 45, 592 (1982).

[45] F. Buff, in Saline Water Conversation Report (U.S. GPO, Washington, DC, 1966). p. 26.

[46] F. Abeles, Thin Solid Films 34, 291 (1976).

[47] E.P. Damm, Jr., J. Electrochem. Soc. 110, 590 (1963).

[48] C.F. Hayes, J. Phys. Chem. 79, 1689 (1975).

[49] M.E. van Leeuwen, Fluid Phase Equilib. 99, 1 (1994).

[50] T.J. Gallagher, Simple Dielectric Liquids: Mobility, Conduction, and Breakdown (Claredon, Oxford, 1975).

[51] B. Groh and S. Dietrich, Phys. Rev. E 53, 2509 (1996).

[52] V.B. Warshavsky and A.K. Shchekin, Colloids Surf., A 148, 283 (1999).

[53] A.K. Shchekin and V.B. Warshavsky, Colloid J. 58, 564 (1996). 